\title{
The Degenerate Case of Boundary Value Problems Associated with Weakly Nonlinear Differential Systems
}

\author{
By \\ Minoru URABE
}

\section{$\S 0$. Introduction}

The present paper is concerned with the boundary value problem of the form:

$$
\begin{gathered}
\frac{d x}{d t}=A(t) x+f(t)+\varepsilon X(t, x, \varepsilon), \\
\sum_{i=0}^{N} L_{i} x\left(t_{i}\right)=l,
\end{gathered}
$$

where $x, f(t)$ and $X(t, x, \varepsilon)$ are vectors, $A(t)$ is a matrix, $\varepsilon$ is a small parameter, $L_{i}(i=0,1,2, \cdots, N)$ are given constant square matrices, $l$ is a given constant vector, and

$$
0=t_{0}<t_{1}<t_{2}<\cdots<t_{N-1}<t_{N}=1 .
$$

As shown in [1], boundary condition (0.2) is of much generality.

Let $\Phi(t)$ be the fundamental matrix of the linear homogeneous system

$$
\frac{d y}{d t}=A(t) y
$$

satisfying the initial condition $\Phi(0)=E$ ( $E$ is the unit matrix). The case where the matrix

$$
G=\sum_{i=0}^{N} L_{i} \Phi\left(t_{i}\right)
$$

Received September 4, 1968. 
is non-singular was discussed already in [1]. Hence the case where matrix $G$ is singular will be discussed in the present paper.

First the boundary value problem with boundary condition (0.2) will be solved for the linear differential system.

$$
\frac{d x}{d t}=A(t) x+f(t)
$$

with the same $A(t)$ as in (0.1).

Next by the use of the above result and an existence theorem of a solution of the equation in Banach spaces established by the author in [2], an existence theorem will be proved for the original boundary value problem $(0.1) \sim(0.2)$. The theorem obtained will be illustrated with an example.

Lastly the theorem obtained will be applied to the boundary value problem associated with the equation of the form

$$
\frac{d \xi}{d t}=\Xi(t, \xi)+\varepsilon \Theta(t, \xi, \varepsilon) \text {. }
$$

The existence theorem obtained in the present paper is based not on the common implicit function theorem but on the existence theorem established by the author in [2]. Hence, in our existence theorem, is given an explicit bound of a small parameter within which the existence of a solution is guaranteed.

\section{§1. Boundary Value Problems Associated with Linear Differential Systems}

1. 1. Lemma concerning linear algebraic equations. We shall state a lemma concerning linear algebraic equations necessary for proving our theorem concerning boundary value problems associated with linear differential systems.

Lemma. Given a system of linear algebraic equations

$$
A x=b,
$$

where $A$ is an $n \times n$ matrix and $x$ and $b$ are both n-dimensional 
vectors. Suppose that the rank of $A$ is $n-m(1 \leqq m \leqq n)$.

Then linear algebraic system (1.1) possesses a solution if and only if

$$
\Delta b=0,
$$

where $\Delta$ is an $m \times n$ matrix whose row vectors are linearly independent vectors $d_{\alpha}(\alpha=1,2, \cdots, m)$ satisfying

$$
d_{\alpha} A=0 .
$$

In case (1.2) holds, any solution of (1.1) can be given by

$$
x=\sum_{\alpha=1}^{m} \kappa_{\alpha} c_{\alpha}+S b,
$$

where $\kappa_{\alpha}(a=1,2, \cdots, m)$ are arbitrary constants, $c_{\alpha}(\alpha=1,2, \cdots, m)$ are $m$ linearly independent column vectors satisfying

$$
A c_{\alpha}=0 \text {, }
$$

and $S$ is an $n \times n$ matrix independent of $b$ such that

$$
A S p=p
$$

for any column vector $p$ satisfying

$$
\Delta p=0 .
$$

The first conclusion of the lemma is well known, but, for the convenience of proving the second conclusion, the complete proof of the lemma will be given below.

Proof. Without loss of generality, we may suppose that matrix $A$ is of the form

$$
A=\left[\begin{array}{ll}
A_{11} & A_{12} \\
A_{21} & A_{22}
\end{array}\right],
$$

where $A_{11}$ is an $(n-m) \times(n-m)$ matrix such that

$$
\operatorname{det} A_{11} \neq 0 \text {. }
$$

Then, since the rank of $A$ is $n-m$, there is an $m \times(n-m)$ matrix $\Delta_{0}$ such that 


$$
\left\{\begin{array}{l}
\Delta_{0} A_{11}+A_{21}=0, \\
\Delta_{0} A_{12}+A_{22}=0 .
\end{array}\right.
$$

Now put $\Delta=\left[\Delta_{1}, \Delta_{2}\right]$, where $\Delta_{1}$ is an $m \times(n-m)$ matrix and $\Delta_{2}$ is an $m \times m$ matrix. Then from (1.8) and (1.3), we see that

$$
\Delta_{1} A_{11}+\Delta_{2} A_{21}=0 .
$$

Since $A_{21}=-\Delta_{0} A_{11}$ from the first of (1.10), we then have

$$
\left(\Delta_{1}-\Delta_{2} \Delta_{0}\right) A_{11}=0,
$$

which, by (1.9), implies

$$
\Delta_{1}=\Delta_{2} \Delta_{0} .
$$

Then we readily see that

$$
\operatorname{det} \Delta_{2} \neq 0 .
$$

In fact, if $\operatorname{det} \Delta_{2}=0$, then there is a non-trivial $m$-dimensional row vector $q$ satisfying $q \Delta_{2}=0$. Then by (1.11) we have $q \Delta_{1}=0$ and hence $q \Delta=0$, which contradicts the assumption on the row vectors of $\Delta$.

Now let us rewrite the given linear system (1.1) as follows:

$$
\left[\begin{array}{ll}
A_{11} & A_{12} \\
A_{21} & A_{22}
\end{array}\right] \cdot\left[\begin{array}{l}
x_{1} \\
x_{2}
\end{array}\right]=\left[\begin{array}{l}
b_{1} \\
b_{2}
\end{array}\right],
$$

that is,

$$
\left\{\begin{array}{l}
A_{11} x_{1}+A_{12} x_{2}=b_{1}, \\
A_{21} x_{1}+A_{22} x_{2}=b_{2} .
\end{array}\right.
$$

If (1.14) possesses a solution $\operatorname{col}\left(x_{1}, x_{2}\right)$, then from (1.10) it must be that

$$
\Delta_{0} b_{1}+b_{2}=0
$$

which, by (1.11) and (1.12), is equivalent to the equality

$$
\Delta_{1} b_{1}+\Delta_{2} b_{2}=0 .
$$

This proves the necessity of condition (1.2).

Now suppose (1.16) holds. Then we have (1.15), consequently 
by (1.10) and (1.15), we have

$$
A_{21} x_{1}+A_{22} x_{2}-b_{2}=-\Delta_{0}\left(A_{11} x_{1}+A_{12} x_{2}-b_{1}\right),
$$

which shows that (1.14) is equivalent to the single equation

$$
A_{11} x_{1}+A_{12} x_{2}=b_{1} \text {. }
$$

By (1.9), the above equation possesses always a solution of the form

$$
\left\{\begin{array}{l}
x_{1}=A_{11}^{-1}\left(b_{1}-A_{12} r\right), \\
x_{2}=r,
\end{array}\right.
$$

where $r$ is an arbitrary $m$-dimensional column vector. This proves the sufficiency of the condition (1.2).

From (1.18), it is seen that if we put

$$
S=\left[\begin{array}{cc}
A_{11}^{-1}-A_{11}^{-1} A_{12} \\
0 & E
\end{array}\right],
$$

then

$$
A S\left[\begin{array}{l}
b_{1} \\
r
\end{array}\right]=\left[\begin{array}{l}
b_{1} \\
b_{2}
\end{array}\right]=\left[\begin{array}{c}
b_{1} \\
-\Delta_{0} b_{1}
\end{array}\right]
$$

for an arbitrary $m$-dimensional vector $r$. Hence, if $r=-\Delta_{0} b_{1}$, that is, $\Delta_{1} b_{1}+\Delta_{2} r=0$, then we have

$$
A S\left[\begin{array}{l}
b_{1} \\
r
\end{array}\right]=\left[\begin{array}{l}
b_{1} \\
r
\end{array}\right]
$$

which proves the existence of a matrix $S$ specified in the lemma. Since $S b$ is a particular solution of the given system (1.1) under condition (1.2), the general solution of (1.1) can be given by (1.4). This completes the proof.

Q. E. D.

If we put

$$
S=\left[\begin{array}{ll}
S_{11} & S_{12} \\
S_{21} & S_{22}
\end{array}\right]
$$

corresponding to the partioning (1.8) of $A$, then by (1.6) and (1.15) the condition for the matrix $S$ can be written as follows: 


$$
\left[\begin{array}{ll}
A_{11} & A_{12} \\
A_{21} & A_{22}
\end{array}\right] \cdot\left[\begin{array}{ll}
S_{11} & S_{12} \\
S_{21} & S_{22}
\end{array}\right] \cdot\left[\begin{array}{c}
p_{1} \\
-\Delta_{0} p_{1}
\end{array}\right]=\left[\begin{array}{c}
p_{1} \\
-\Delta_{0} p_{1}
\end{array}\right],
$$

where $p_{1}$ is an arbitrary $(n-m)$-dimensional column vector. By (1.10), the above condition is equivalent to the condition

$$
\left[A_{11}\left(S_{11}-S_{12} \Delta_{0}\right)+A_{12}\left(S_{21}-S_{22} \Delta_{0}\right)\right] p_{1}=p_{1} .
$$

Since $p_{1}$ is arbitrary, the above condition is equivalent to the condition

$$
A_{11}\left(S_{11}-S_{12} \Delta_{0}\right)+A_{12}\left(S_{21}-S_{22} \Delta_{0}\right)=E,
$$

where $E$ is the $(n-m) \times(n-m)$ unit matrix. Since $\Delta_{0}=-A_{21} A_{11}^{-1}$ by the first of (1.10), we readily see that the above condition can be written as follows:

$$
\left[A_{11}, A_{12}\right] \cdot\left[\begin{array}{ll}
S_{11} & S_{12} \\
S_{21} & S_{22}
\end{array}\right] \cdot\left[\begin{array}{c}
A_{11} \\
A_{21}
\end{array}\right]=A_{11}
$$

This is the sole condition necessary and sufficient for the matrix $S$. Evidently matrix $S$ of the form (1.19) satisfies (1.22), but matrix $S$ of the form $\left[\begin{array}{cc}A_{11}^{-1} & 0 \\ 0 & 0\end{array}\right]$ also satisfies (1.22). It is thus clear that the matrix $S$ specified in the lemma is not unique.

1.2. Theorem concerning boundary value problems associated with linear differential systems.

Theorem 1. Let

$$
\frac{d x}{d t}=A(t) x+f(t)
$$

be a given n-dimensional linear differential system where $A(t)$ is an $n \times n$ matrix continuous on the interval $I[0,1]$ and $f(t)$ is an $n$-dimensional vector continuous on $I$.

Let $\Phi(t)$ be the fundamental matrix of the corresponding homogeneous system

$$
\frac{d y}{d t}=A(t) y
$$

satisfying the initial condition $\Phi(0)=E$, and suppose that the rank of the matrix 


$$
G=\sum_{i=0}^{N} L_{i} \Phi\left(t_{i}\right)
$$

is $n-m(1 \leqq m \leqq n)$ for

$$
0=t_{0}<t_{1}<t_{2}<\cdots<t_{N-1}<t_{N}=1
$$

and given square matrices $L_{i}(i=0,1,2, \cdots, N)$.

Then the given system (1.23) possesses a solution satisfying the boundary condition

$$
\sum_{i=0}^{N} L_{i} x\left(t_{i}\right)=l
$$

if and only if

$$
\Delta l-\Delta \sum_{i=0}^{N} L_{i} \Phi\left(t_{i}\right) \int_{0}^{t_{i}} \Phi^{-1}(t) f(t) d t=0,
$$

where $\Delta$ is an $m \times n$ matrix whose row vectors are linearly independent vectors $d_{\alpha}(\alpha=1,2, \cdots, m)$ satisfying

$$
d_{\alpha} G=0 \text {. }
$$

In case (1.27) is valid for given $l$ and $f(t)$, any solution of (1.23) satisfying boundary condition (1.26) can be given by

$$
x(t)=\sum_{\alpha=1}^{m} \kappa_{\alpha} \phi_{\alpha}(t)+\Phi(t) S l+\int_{0}^{1} H(t, s) f(s) d s,
$$

where $\kappa_{\alpha}(\alpha=1,2, \cdots, m)$ are arbitrary constants, $\phi_{\alpha}(t)(\alpha=1,2, \cdots, m)$ are $m$ linearly independent solutions of (1.24) satisfying the boundary condition

$$
\sum_{i=0}^{N} L_{i} y\left(t_{i}\right)=0,
$$

$S$ is a matrix independent of $f(t)$ and $l$ such that

$$
G S p=p
$$

for any $n$-dimensional vector $p$ satisfying

$$
\Delta p=0,
$$

and $H(t, s)$ is the piece-wise continuous matrix such that, for $t_{k-1} \leqq s<t_{k} \quad(k=1,2, \cdots, N)$, 


$$
H(t, s)= \begin{cases}\Phi(t)\left[E-S \sum_{i=k}^{N} L_{i} \Phi\left(t_{i}\right)\right] \Phi^{-1}(s) & \text { if } s<t, \\ -\Phi(t) S \sum_{i=k}^{N} L_{i} \Phi\left(t_{i}\right) \cdot \Phi^{-1}(s) & \text { if } s \geqq t .\end{cases}
$$

Proof. Any solution of (1.23) can be written as

$$
x=x(t)=\emptyset(t) c+\Phi(t) \int_{0}^{t} \Phi^{-1}(s) f(s) d s,
$$

where $c$ is a constant vector. The solution (1.34) satisfies boundary condition (1.26) if and only if

$$
\sum_{j=0}^{N} L_{i} \Phi\left(t_{i}\right) \cdot c+\sum_{i=0}^{N} L_{i} \Phi\left(t_{i}\right) \int_{0}^{t_{i}} \Phi^{-1}(s) f(s) d s=l,
$$

that is,

$$
G c=l-\sum_{i=0}^{N} L_{i} \Phi\left(t_{i}\right) \int_{0}^{t_{i}} \Phi^{-1}(s) f(s) d s .
$$

Now by assumption the rank of $G$ is $n-m$. Therefore by the lemma in 1.1 the constant vector $c$ satisfying (1.35) exists if and only if (1.27) holds. This proves the first conclusion of the theorem.

When (1.27) holds, by the lemma of 1.1 the constant vector $c$ satisfying (1.35) can be given by

$$
c=\sum_{\alpha=1}^{m} \kappa_{\alpha} c_{\alpha}+S\left[l-\sum_{i=0}^{N} L_{i} \Phi\left(t_{i}\right) \int_{0}^{t_{i}} \Phi^{-1}(s) f(s) d s\right],
$$

where $\kappa_{\alpha}(\alpha=1,2, \cdots, m)$ are arbitrary constants, $c_{\alpha}(\alpha=1,2, \cdots, m)$ are $m$ linearly independent column vectors satisfying

$$
G c_{\alpha}=0 \text {, }
$$

and $S$ is an $n \times n$ matrix independent of the right member of (1.35) such that (1.31) holds for any vector $p$ satisfying (1.32). Put

$$
\varpi(t) c_{\alpha}=\phi_{\alpha}(t) \quad(\alpha=1,2, \cdots, m),
$$

then evidently $\phi_{\alpha}(t)(\alpha=1,2, \cdots, m)$ are linearly independent and satisfy (1.24). Moreover by (1.25) and (1.37), it holds that

$$
\begin{aligned}
\sum_{i=0}^{N} L_{i} \phi_{\alpha}\left(t_{i}\right) & =\sum_{i=0}^{N} L_{i} \Phi\left(t_{i}\right) \cdot c_{\alpha} \\
& =G c_{\alpha}=0 .
\end{aligned}
$$


Now substitute (1.36) into (1.34), then making use of (1.38) and (1.33), we have successively

$$
\begin{aligned}
x(t)= & \sum_{\alpha=1}^{m} \kappa_{\alpha} \phi_{\alpha}(t)+\Phi(t) S l \\
& -\Phi(t) S \sum_{i=0}^{N} L_{i} \Phi\left(t_{i}\right) \int_{0}^{t_{i}} \Phi^{-1}(s) f(s) d s \\
& +\Phi(t) \int_{0}^{t} \Phi^{-1}(s) f(s) d s \\
= & \sum_{\alpha=1}^{m} \kappa_{\alpha} \phi_{\alpha}(t)+\Phi(t) S l \\
& -\Phi(t) S \sum_{k=1}^{N}\left[\sum_{i=k}^{N} L_{i} \Phi\left(t_{i}\right) \int_{t_{k-1}}^{t_{k}} \Phi^{-1}(s) f(s) d s\right] \\
& +\Phi(t) \int_{0}^{t} \Phi^{-1}(s) f(s) d s \\
= & \sum_{\alpha=1}^{m} \kappa_{\alpha} \phi_{\alpha}(t)+\Phi(t) S l+\int_{0}^{1} H(t, s) f(s) d s .
\end{aligned}
$$

This completes the proof.

Q. E. D.

As was mentioned before, the matrix $S$ specified in Theorem 1 is not unique, but after it has been chosen once in any way, it will be fixed throughout the succeeding discussions. Hence we may suppose that $H(t, s)$ is a definite matrix and it depends only on matrices $A(t)$ and $L_{i}(i=0,1,2, \cdots, N)$.

Remark. For an arbitrary $n$-dimensional continuous vector function $f(t)$, put

$$
u(t)=\int_{0}^{1} H(t, s) f(s) d s .
$$

By (1.40), the above equality means that

$$
\begin{aligned}
u(t)= & -\Phi(t) S \sum_{i=0}^{N} L_{i} \Phi\left(t_{i}\right) \int_{0}^{t_{i}} \Phi^{-1}(s) f(s) d s \\
& +\Phi(t) \int_{0}^{t} \Phi^{-1}(s) f(s) d s .
\end{aligned}
$$

Hence it is clear that

$$
\frac{d u(t)}{d t}=A(t) u(t)+f(t)
$$


From (1.42) we have also

$$
\begin{aligned}
\sum_{i=0}^{N} L_{i} u\left(t_{i}\right)= & -G S \sum_{i=0}^{N} L_{i} \Phi\left(t_{i}\right) \int_{0}^{t_{i}} \Phi^{-1}(s) f(s) d s \\
& +\sum_{i=0}^{N} L_{i} \Phi\left(t_{i}\right) \int_{0}^{t_{i}} \Phi^{-1}(s) f(s) d s \\
= & (E-G S) \sum_{i=0}^{N} L_{i} \Phi\left(t_{i}\right) \int_{0}^{t_{i}} \Phi^{-1}(s) f(s) d s .
\end{aligned}
$$

If

$$
\Delta \sum_{i=0}^{N} L_{i} \Phi\left(t_{i}\right) \int_{0}^{t_{i}} \Phi^{-1}(s) f(s) d s=\Delta l^{\prime}
$$

for some $n$-dimensional vector $l^{\prime}$, then we have

$$
\Delta\left[\sum_{i=0}^{N} L_{i} \Phi\left(t_{i}\right) \int_{0}^{t_{i}} \Phi^{-1}(s) f(s) d s-l^{\prime}\right]=0
$$

and hence

$$
\begin{gathered}
G S\left[\sum_{i=0}^{N} L_{i} \Phi\left(t_{i}\right) \int_{0}^{t_{i}} \Phi^{-1}(s) f(s) d s-l^{\prime}\right] \\
=\sum_{i=0}^{N} L_{i} \Phi\left(t_{i}\right) \int_{0}^{t_{i}} \Phi^{-1}(s) f(s) d s-l^{\prime} .
\end{gathered}
$$

Then from (1.44) we have

$$
\sum_{i=0}^{N} L_{i} u\left(t_{i}\right)=(E-G S) l^{\prime}
$$

Now $u(t)$ defined by (1.41) is continuous as seen from (1.42), therefore formula (1.41) defines a linear mapping in the space of continuous vector functions defined on $I$. Since the matrix $H(t, s)$ is dependent only on matrices $A(t)$ and $L_{i}(i=1,2, \cdots, N)$, the mapping defined by (1.41) will be called hereafter the $H$-mapping corresponding to matrices $A(t)$ and $L_{i}(i=0,1,2, \cdots, N)$.

\section{§2. An Existence Theorem of a Solution of the Equation in Banach Spaces}

We shall state a thecrem necessary for proving our theorem concerning the given boundary value problem $(0.1) \sim(0.2)$.

Theorem 2. Let $F(x)$ be a function mapping an open set $D$ of a Banach space $B_{1}$ into another Banach space $B_{2}$ which may co- 
incide with $B_{1}$. Suppose that the Frechet derivative $J(x)$ of $F(x)$ is continuous on $D$ and the equation

$$
F(x)=0
$$

possesses an approximate solution $x=\bar{x} \in D$, for which there are an additive operator $\hat{J}$ mapping $B_{1}$ into $B_{2}$, a positive number $\delta$, and a non-negative number $k<1$ such that

$$
\widehat{J} \text { possesses an inverse linear operator } \widehat{J}^{-1} \text {, }
$$

Here $r(\geqq 0)$ and $M(>0)$ are numbers such that

$$
\begin{gathered}
\|F(\bar{x})\| \leqq r, \\
\left\|\widehat{J}^{-1}\right\| \leqq M .
\end{gathered}
$$

Then the given equation (2.1) possesses one and only one solution $x=\hat{x}$ in $D_{\delta}$ and moreover, for $x=\hat{x}, J^{-1}(\hat{x})$ exists and

$$
\|\hat{x}-\bar{x}\| \leqq M r /(1-k) .
$$

This theorem has been already proved in [2] except the conclusion on the existence of $J^{-1}(\hat{x})$. Hence only the outline of the proof will be given here.

Proof. Putting $\bar{x}=x_{0}$, consider the Newton iterative process

$$
x_{n+1}=x_{n}-\widehat{J}^{-1} F\left(x_{n}\right) \quad(n=0,1,2, \cdots) .
$$

Then making use of the equality

$$
\begin{aligned}
& x_{n+1}-x_{n}=\left(x_{n}-x_{n-1}\right)-\widehat{J}^{-1}\left[F\left(x_{n}\right)-F\left(x_{n-1}\right)\right] \\
= & \widehat{J}^{-1} \int_{0}^{1}\left\{\widehat{J}-J\left[x_{n-1}+\theta\left(x_{n}-x_{n-1}\right)\right]\right\}\left(x_{n}-x_{n-1}\right) d \theta,
\end{aligned}
$$

by the induction we have

$$
\begin{aligned}
& \left\|x_{n+1}-x_{n}\right\| \leqq k^{n}\left\|x_{1}-x_{0}\right\|, \\
& \left\|x_{n+1}-x_{0}\right\| \leqq M r /(1-k) \leqq \delta \quad(n=0,1,2, \cdots) .
\end{aligned}
$$


The above inequalities show that the sequence $\left\{x_{n}\right\}$ is a fundamental sequence in $D_{\delta} \subset B_{1}$. Hence we have

$$
\hat{x}=\lim _{n \rightarrow \infty} x_{n} \in D_{\delta}
$$

and moreover, by (2.12),

$$
\|\hat{x}-\bar{x}\| \leqq M r /(1-k) .
$$

We can see easily that $\hat{x}$ is a solution of equation (2.1). Thus we see the existence of a solution of (2.1) in $D_{\delta}$ and the validity of inequality (2.8). The uniqueness of a solution in $D_{\delta}$ can be proved easily if we use an equality similar to (2.10).

Finally let us prove the existence of $J^{-1}(\hat{x})$. Write $J(\hat{x})$ in the following form:

$$
\begin{aligned}
J(\hat{x}) & =\widehat{J}+[J(\hat{x})-\widehat{J}] \\
& =\widehat{J}\left\{e+\widehat{J}^{-1}[J(\hat{x})-\widehat{J}]\right\},
\end{aligned}
$$

where $e$ is the identical operator. In (2.15), by (2.4) and (2.7)

$$
\left\|\widehat{J}^{-1}[J(\hat{x})-\widehat{J}]\right\| \leqq M \cdot \frac{k}{M}=k<1 .
$$

Hence by the well-known theorem we see that the operator $e+\widehat{J}^{-1}[J(\hat{x})$ $-\widehat{J}]$ possesses an inverse. From this readily follows the existence of $J^{-1}(\hat{x})$. This completes the proof.

Q. E. D.

As stated in [2], conditions $(2.3) \sim(2.5)$ are related with the accuracy of the given approximate solution $x=\bar{x}$, in other words, they prescribe the accuracy of the approximate solution which allows one to assure the existence of an exact solution and the related conclusions of the theorem.

\section{§3. Boundary Value Problems Associated with Weakly Nonlinear Differential Systems}

In what follows, we shall denote by the symbol $\|\cdots\|$ the Euclidean norm of vectors and the corresponding norm of matrices. For continuous vector functions defined on the interval $I[0,1]$, we shall use the uniform 
norm and d:act: it by the symbol $\|\cdots\|_{n}$. Namely let $f(t)$ be an arbitrary vector furction continuous on $I$, then $\|f(t)\|_{n}$ will mean $\sup _{t \in I}\|f(t)\|$, where $\|f(t)\|$ is the Euclidean norm of vector $f(t)$.

In the present paragraph, our concern is about the boundary value problem of the following form:

$$
\begin{aligned}
& \frac{d x}{d t}=A(t) x+f(t)+\varepsilon X(t, x, \varepsilon), \\
& \sum_{i=0}^{N} L_{i} x\left(t_{i}\right)=l,
\end{aligned}
$$

where $x, f(t)$ and $X(t, x, \varepsilon)$ are $n$-dimensional veztors, $A(t)$ is an $n \times n$ matrix, $\varepsilon$ is a parameter, $L_{i}(i=0,1,2, \cdots, N)$ are given $n \times n$ matrices, $l$ is a given $n$-dimensional vector, and

$$
0=t_{0}<t_{1}<t_{2}<\cdots<t_{N-1}<t_{N}=1 \text {. }
$$

3.1. Setting of the problem and assumptions. In (3.1) we assume that

$$
\begin{aligned}
& A(t) \text { and } f(t) \text { are continuous on } I, \\
& X(t, x, \varepsilon) \in C_{x, \varepsilon}^{1} \text { for }(t, x) \in \Omega,|\varepsilon| \leqq \varepsilon_{0}, \\
& \Psi(t, x, \varepsilon) \in C_{x, \varepsilon}^{1} \text { for }(t, x) \in \Omega,|\varepsilon| \leqq \varepsilon_{0},
\end{aligned}
$$

where $\varepsilon_{0}$ is a positive number, $\Psi(t, x, \varepsilon)$ is the Jacobian matrix of $X(t, x, \varepsilon)$ with respect to $x$, and $\Omega$ is the domain of the $t x$-space intercepted by two hyperplanes $t=0$ and $t=1$ such that every section of $\Omega$ by an arbitrary hyperplane $t=\tau(0 \leqq \tau \leqq 1)$ is a non-empty open set of the $x$-space.

We assume further that the rank of the matrix

$$
G=\sum_{i=0}^{N} L_{i} \Phi\left(t_{i}\right)
$$

is $n-m(1 \leqq m \leqq n)$, where $\Phi(t)$ is the fundamental matrix of the linear homogeneous system

$$
\frac{d y}{d t}=A(t) y
$$

satisfying the initial condition $\Phi(0)=E$. 
By the latter assumption, as stated in Theorem 1, there are $m$ linearly independent solutiors $\phi_{\alpha}(t)(\alpha=1,2, \cdots, m)$ of (3.4) satisfying the boundary condition

$$
\sum_{i=0}^{N} L_{i} y\left(t_{i}\right)=0
$$

and $m$ linearly independent row vectors $d_{\alpha}(\alpha=1,2, \cdots, m)$ satisfying

$$
d_{\alpha} G=0 .
$$

For these $\phi_{\alpha}(t)$ and $d_{\alpha}$, by the normalization we may suppose without loss of generality that

$$
\begin{aligned}
& \sum_{\alpha=1}^{m}\left\|\phi_{\alpha}\right\|_{n}^{2}=1, \\
& \sum_{\alpha=1}^{m}\left\|d_{\alpha}\right\|^{2}=1 .
\end{aligned}
$$

Now by Theorem 1 our boundary value problem (3.1) (3.2) possesses a solution for $\varepsilon=0$ if and only if

$$
\Delta l-\Delta \sum_{i=0}^{N} L_{i} \Phi\left(t_{i}\right) \int_{0}^{t_{i}} \Phi^{-1}(s) f(s) d s=0,
$$

where $\Delta$ is the matrix whose row vectors are $d_{\alpha}(\alpha=1,2, \cdots, m)$. Then, when (3.9) is valid for $f(t)$ and $l$, under what condition does our boundary value problem $(3.1) \sim(3.2)$ possess a solution for small $|\varepsilon|>0$ ?

Suppose that for small $|\varepsilon|>0$, our boundary value problem (3.1) $\sim(3.2)$ has possessed a solution $x=x(t)$ such that $(t, x(t)) \in \Omega$. Then by Theorem 1 we have

$$
\begin{aligned}
x(t) & =\sum_{\alpha=1}^{m} \kappa_{\alpha} \phi_{\alpha}(t)+\Phi(t) S l \\
& +\int_{0}^{1} H(t, s)\{f(s)+\varepsilon X[s, x(s), \varepsilon]\} d s,
\end{aligned}
$$

$$
\Delta l-\Delta \sum_{i=0}^{N} L_{i} \Phi\left(t_{i}\right) \int_{0}^{t_{i}} \Phi^{-1}(s)\{f(s)+\varepsilon X[s, x(s), \varepsilon]\} d s=0,
$$

where $\kappa_{\alpha}(\alpha=1,2, \cdots, m)$ are arbitrary constants, $S$ is the matrix specified as in Theorem 1 corresponding to matrix $G$, and $H(t, s)$ is the 
matrix of the $H$-mapping corresponding to matrices $A(t)$ and $L_{i}$ ( $i$ $=0,1,2, \cdots, N)$. Since $\varepsilon \neq 0$, condition (3.11) is equivalent, by (3.9), to the condition

$$
\Delta \sum_{i=0}^{N} L_{i} \Phi\left(t_{i}\right) \int_{0}^{t_{t}} \Phi^{-1}(s) X[s, x(s), \varepsilon] d s=0 .
$$

If we substitute (3.10) into (3.12), then we have

$$
\begin{aligned}
& \Delta \sum_{i=0}^{N} L \emptyset_{i}\left(t_{i}\right) \int_{0}^{t_{i}} \Phi^{-1}(s) X\left[s, \sum_{\alpha=1}^{m} \kappa_{\alpha} \phi_{\alpha}(s)+\Phi(s) S l\right. \\
& \left.\quad+\int_{0}^{1} H(s, \sigma) f(\sigma) d \sigma+\varepsilon \int_{0}^{1} H(s, \sigma) X[\sigma, x(\sigma), \varepsilon] d \sigma, \varepsilon\right] d s=0,
\end{aligned}
$$

which, as $\varepsilon \rightarrow 0$, tends to the equality

$$
\begin{gathered}
\Delta \sum_{i=0}^{N} L_{i} \Phi\left(t_{i}\right) \int_{0}^{t_{i}} \Phi^{-1}(s) X\left[s, \sum_{\alpha=1}^{m} \kappa_{\alpha} \phi_{\alpha}(s)+\Phi(s) S l\right. \\
\left.\quad+\int_{0}^{1} H(s, \sigma) f(\sigma) d \sigma, 0\right] d s=0 .
\end{gathered}
$$

Thus we see that if there do not exist $\kappa_{\alpha}(\alpha=1,2, \cdots, m)$ satisfying (3. 14), then our boundary value problem (3.1) (3.2) cannot possess a solution for small $|\varepsilon|>0$.

The problem is thus to examine whether or not our boundary value problem (3.1) (3.2) possesses a solution for small $|\varepsilon|>0$ when (3.9) is valid and in addition there exist $\kappa_{\alpha}=\kappa_{\alpha}^{0}(\alpha=1,2, \cdots, m)$ satisfying (3.14).

Our setting of the problem thus includes the assumptions that (3.9) is valid for given vectors $f(t)$ and $l$, and that there exist $\kappa_{\alpha}=\kappa_{\alpha}^{0}(\alpha=1,2, \cdots, m)$ satisfying (3.14) such that the graph of the function

$$
x=x_{0}(t)=\sum_{\alpha=1}^{m} \kappa_{\alpha}^{0} \phi_{\alpha}(t)+\Phi(t) S l+\int_{0}^{1} H(t, s) f(s) d s
$$

lies in domain $\Omega$ for $0 \leqq t \leqq 1$.

3. 2. Various relating constants. Our theorem concerning boundary value problem $(3.1) \sim(3.2)$ necessitates to introduce various constants. Hence the definitions of these constants will be given in advance in the present section. 
$1^{\circ}$ Constant $\delta_{0}>0$. Constant $\delta_{0}$ is a positive number such that

$$
\Omega_{0}=\left\{(t, x) \mid\left\|x-x_{0}(t)\right\| \leqq \delta_{0}, t \in I\right\} \subset \Omega
$$

for $x_{0}(t)$ given by (3.15). The existence of such positive number $\delta_{0}$ follows from the definition of domain $\Omega$.

$2^{\circ}$ Constants $K_{i}>0(i=0,1,2,3,4)$. Constants $K_{0}, K_{1}$ and $K_{2}$ are positive numbers such that

$$
\left\{\begin{array}{l}
\|X(t, x, \varepsilon)\| \leqq K_{0}, \\
\|\Psi(t, x, \varepsilon)\| \leqq K_{1}, \\
\left\|\frac{\partial X}{\partial \varepsilon}(t, x, \varepsilon)\right\| \leqq K_{2}
\end{array}\right.
$$

for any $(t, x) \in \Omega_{0}$ and any $\varepsilon$ satisfying $|\varepsilon| \leqq \varepsilon_{0}$. Constants $K_{3}$ and $K_{4}$ are the positive numbers such that

$$
\left\|\Psi\left(t, x^{\prime}, \varepsilon^{\prime}\right)-\Psi\left(t, x^{\prime \prime}, \varepsilon^{\prime \prime}\right)\right\| \leqq K_{3}\left\|x^{\prime}-x^{\prime \prime}\right\|+K_{4}\left|\varepsilon^{\prime}-\varepsilon^{\prime \prime}\right|
$$

for any $\left(t, x^{\prime}\right),\left(t, x^{\prime \prime}\right) \in \Omega_{0}$ and any $\varepsilon^{\prime}, \varepsilon^{\prime \prime}$ satisfying $\left|\varepsilon^{\prime}\right|,\left|\varepsilon^{\prime \prime}\right| \leqq \varepsilon_{0}$. The existence of these positive constants is evident from the continuity or the smoothness of the related functions and the compactness of the domain of definition.

$3^{\circ}$ Constant $H_{0}>0 . \quad H_{0}$ is a number not small than the uniform norm of the $H$-mapping corresponding to matrices $A(t)$ and $L_{i}(i=0$, $1,2, \cdots, N)$, that is, a number such that

$$
\left\|\int_{0}^{1} H(t, s) \psi(s) d s\right\|_{n} \leqq H_{0} \cdot\|\psi\|_{n}
$$

for any vector function $\psi(t)$ continuous on $I$, where $H(t, s)$ is the matrix of the $H$-mapping corresponding to $A(t)$ and $L_{i}(i=0,1,2, \cdots$, $N)$. Number $H_{0}$ is always positive, because otherwise $H_{0}=0$ and hence

$$
\int_{0}^{1} H(t, s) \psi(s) d s=0
$$

for any $\psi(t) \in C[I]$, which is a contradiction since (3.20) implies $\psi(t)=0$ by Remark in $\mathbf{1 . 2}$.

$4^{\circ}$ Positive constants $L, V$ and $W$. Constant $L$ is the number such that 


$$
\sum_{i=0}^{N}\left\|L_{\imath}\right\|=L
$$

If $L=0$, then $L=0(i=0,1,2, \cdots, N)$ and in such a case boundary condition (3.2) loses its proper meaning. Hence we may suppose naturally that $L>0$.

Constants $V$ and $W$ are the numbers such that

$$
\sup _{t \in I}\|\emptyset(t)\|=V, \quad \sup _{t \subseteq I}\left\|\Phi^{-1}(t)\right\|=W .
$$

Since $\mathscr{D}(t)$ is non-singular for any $t \in I$, it is evident that $V, W>0$.

$5^{\circ}$ Constant $K>0$. Corsider the equation

$$
\sum_{\alpha=1}^{m} \kappa_{\alpha} \phi_{\alpha}(t)=v(t)
$$

then $K$ is a positive number such that

$$
\|\kappa\| \leq K\|v\|_{n},
$$

where $\kappa$ is an $m$-dimensional vector whose components are $\kappa_{\alpha}(\alpha=1,2$, $\cdots, m)$.

Number $\kappa$ can be obtained in a following way.

First, according to the definition of functions $\phi_{\alpha}(t)(\alpha=1,2, \cdots, m)$, rewrite (3.23) in the following form:

$$
\Phi(t) \sum_{\alpha=1}^{m} \kappa_{\alpha} c_{\alpha}=v(t),
$$

where $c_{\alpha}(\alpha=1,2, \cdots, m)$ are $m$ linearly independent vectors satisfying

$$
G c_{\alpha}=0 \text {. }
$$

Next, take $n-m$ linearly independent vectors $c_{\nu}(\nu=m+1, m+2, \cdots, n)$ so that $c_{1}, c_{2}, \cdots, c_{m}, c_{m+1}, \cdots, c_{n}$ may be all linearly independent. Then, if we denote by $C$ the matrix whose column vectors are $c_{i}(i=1,2, \cdots$, $n)$, we can write (3.25) as follows:

$$
\Phi(t) C \cdot \operatorname{col}\left(\kappa_{1}, \kappa_{2}, \cdots, \kappa_{m}, 0, \cdots, 0\right)=v(t),
$$

from which readily follows

$$
\operatorname{col}\left(\kappa_{1}, \kappa_{2}, \cdots, \kappa_{m}, 0, \cdots, 0\right)=C^{-1} \Phi^{-1}(t) v(t) .
$$

Hence we have 


$$
\|\kappa\| \leqq\left\|C^{-1}\right\| \cdot\left\|\Phi^{-1}(t)\right\| \cdot\|v(t)\| \leqq\left\|C^{-1}\right\| \cdot W \cdot\|v\|_{n} .
$$

Thus we may take $K$ so that

$$
K=W \cdot\left\|C^{-1}\right\| \text {. }
$$

3. 3. Theorem concerning boundary value problem (3.1) (3.2).

Theorem 3. For boundary value problem (3.1) (3.2), assume that the appearing functions have the smoothness mentioned in 3.1 and that the rank of matrix $G$ defined by (3.3) is $n-m(1 \leqq m \leqq n)$. If (3.9) is valid for $f(t)$ and $l$ and there exist $\kappa_{\alpha}=\kappa_{\alpha}^{0}(\alpha=1,2, \cdots, m)$ satisfying (3.14) such that the graph of function $x=x_{0}(t)$ given by (3.15) lies in $\Omega$ and the Jacobian matrix $J_{2}$ of the left member of (3.14) with respect to $\kappa_{\alpha}(\alpha=1,2, \cdots, m)$ is non-singular for $\kappa_{\alpha}=\kappa_{\alpha}^{0}$ $(\alpha=1,2, \cdots, m)$, then given boundary value problem (3.1) (3.2) possesses an isolated solution ${ }^{1)} x=\hat{x}(t)$ for any $\varepsilon$ such that

$$
0<|\varepsilon| \leqq \varepsilon_{1}
$$

where

(3. 28) $\varepsilon_{1}=\min \left\{\varepsilon_{0}, \delta_{0}\left[\left(1+\frac{M}{1-k}\right) H_{0} K_{0}+\frac{L M V W}{1-k}\left(H_{0} K_{0} K_{1}+K_{2}\right)\right]^{-1}\right.$,

$$
\begin{aligned}
& \frac{k}{L M V W}\left[\frac{M K_{3}}{1-k}\left[H_{0} K_{0}+L V W\left(H_{0} K_{0} K_{1}+K_{2}\right)\right]\right. \\
& \left.+\left(H_{0} K_{0} K_{3}+K_{4}\right)\right]^{-1}, \\
& \left.\frac{k}{M H_{0} K_{1}}\left(1+L V W K_{1}\right)^{-1}\right\} .
\end{aligned}
$$

In (3.28), $k$ is an arbitrary positive number smaller than 1 and

$$
M=\max \left[1,2\left\|J_{2}^{-1}\right\|\right] \text {. }
$$

1) A solution $x=x(t)$ of the boundary value problem

$$
\begin{aligned}
& \frac{d x}{d t}=X(t, x) \\
& \sum_{i=0}^{N} L_{i} x\left(t_{i}\right)=l
\end{aligned}
$$

is called to be isolated if the matrix $\sum L_{i} \Phi_{1}\left(t_{i}\right)$ is non-singular, where $\Phi_{1}(t)$ is the fundamental matrix of the first variation equation of (i) with respect to the solution $x=x(t)$ satisfying the initial condition $\emptyset_{1}(0)=E$. For details, see [1]. 
For the isolated solution $x=\hat{x}(t)$, it holds that

$$
\left\|\hat{x}-x_{0}\right\|_{n} \leqq \frac{M}{1-k}\left[H_{0} K_{0}+L V W\left(H_{0} K_{0} K_{1}+K_{2}\right)\right]|\varepsilon|
$$

and moreover the solution of boundary value problem (3.1) (3.2) is unique in the region

$$
\left\|x-x_{0}\right\|_{n} \leqq \frac{1}{1+K}\left(\delta-|\varepsilon| H_{0} K_{0} K\right)
$$

for $\varepsilon$ such that

$$
0<|\varepsilon|<\min \left(\varepsilon_{1}, \frac{\delta}{H_{0} K_{0} K}\right),
$$

where

$$
\delta=\min \left\{\delta_{0}-\varepsilon_{1} H_{0} K_{0}, \frac{1}{K_{3}}\left[\frac{k}{L M V W}-\varepsilon_{1}\left(H_{0} K_{0} K_{3}+K_{4}\right)\right]\right\} .
$$

Proof. Let $x=x(t)$ ke an arbitrary solution of boundary value problem (3.1) (3.2) such that $(t, x(t)) \in \Omega$. Then by Theorem 1 we have (3.10) and (3.11). However by the assumption we have (3.9). Hence, if we replace $\kappa_{\alpha}$ by $\kappa_{\alpha}^{0}+\kappa_{\alpha}$, then for $\varepsilon \neq 0$, using (3.15), we have:

$$
\left\{\begin{array}{l}
x(t)=x_{0}(t)+\sum_{\alpha=1}^{m} \kappa_{\alpha} \phi_{\alpha}(t)+\varepsilon \int_{0}^{1} H(t, s) X[s, x(s), \varepsilon] d s, \\
\Delta \sum_{i=0}^{N} L_{i} \Phi\left(t_{i}\right) \int_{0}^{t_{s}} \Phi^{-1}(s) X[s, x(s), \varepsilon] d s=0,
\end{array}\right.
$$

which is evidently equivalent to the system of equations

$$
\left\{\begin{array}{c}
F_{1}(x, \kappa ; \varepsilon) \stackrel{\text { def }}{=} x(t)-x_{0}(t)-\sum_{\alpha=1}^{m} \kappa_{\alpha} \phi_{\alpha}(t) \\
-\varepsilon \int_{0}^{1} H(t, s) X[s, x(s), \varepsilon] d s=0, \\
F_{2}(x, \kappa ; \varepsilon) \stackrel{\text { def }}{=} \Delta \sum_{i=0}^{N} L_{\imath} \Phi\left(t_{i}\right) \int_{0}^{t_{s}} \Phi^{-1}(s) X\left[s, x_{0}(s)\right. \\
\left.+\sum_{\alpha=1}^{m} \kappa_{\alpha} \phi_{\alpha}(s)+\varepsilon \int_{0}^{1} H(s, \sigma) X[\sigma, x(\sigma), \varepsilon] d \sigma, \varepsilon\right] d s=0,
\end{array}\right.
$$

where $\kappa$ is an $m$-dimensional vector whose components are $\kappa_{\alpha}(\alpha=1,2$, $\cdots, m)$. From the derivation of (3.34), it is clear that for $\varepsilon \neq 0,\{x(t), \kappa\}$ with some $\kappa$ is a solution of (3.35) if and only if $x(t)$ is a solution 
of boundary value problem (3.1) (3.2).

Put

$$
F(x, \kappa ; \varepsilon)=\left\{F_{1}(x, \kappa ; \varepsilon), F_{2}(x, \kappa ; \varepsilon)\right\},
$$

then equation (3.35) can be written as

$$
F(x, \kappa ; \varepsilon)=0 \text {, }
$$

which can be regarded as an equation in a Banach space with unknown $\{x(t), \kappa\}$. Moreover, for small $|\varepsilon|$,

$$
F_{1}\left(x_{0}, 0 ; \varepsilon\right)=-\varepsilon \int_{0}^{1} H(t, s) X\left[s, x_{0}(s), \varepsilon\right] d s
$$

is small and

$$
\begin{aligned}
& F_{2}\left(x_{0}, 0 ; \varepsilon\right)=\Delta \sum_{i=0}^{N} L_{i} \Phi\left(t_{i}\right) \int_{0}^{t_{t}} \Phi^{-1}(s) \\
& \quad \times X\left[s, x_{0}(s)+\varepsilon \int_{0}^{1} H(s, \sigma) X\left[\sigma, x_{0}(\sigma), \varepsilon\right] d \sigma, \varepsilon\right] d s
\end{aligned}
$$

is also small as seen from (3.15) and (3.14). Hence $\left\{x_{0}(t), 0\right\}$ is an approximate solution of (3.36). This suggests the application of Theorem 2 to equation (3.36) for assuring the existence of its solution. Needless to say, the existence of a solution of (3.36) implies the existence of a solution of our boundary value problem (3.1) (3.2).

Let us define the norms of $\{x(t), \kappa\}$ and $F(x, \kappa ; \varepsilon)$ respectively by

$$
\left\{\begin{array}{l}
\|\{x(t), \kappa\}\|=\|x(t)\|_{n}+\|\kappa\|, \\
\|F(x, \kappa ; \varepsilon)\|=F_{1}(x, \kappa ; \varepsilon)\left\|_{n}+\right\| F_{2}(x, \kappa ; \varepsilon) \|,
\end{array}\right.
$$

and let us examine the conditions of Theorem 2 one by one.

(i) The domain of definition of $F(x, \kappa ; \varepsilon)$. For $\left\|x-x_{0}\right\|_{n} \leqq \delta_{0}$ and $|\varepsilon| \leqq \varepsilon_{1}$, by (3.19), (3.17) and (3.7), we have

$$
\begin{aligned}
& \left\|\sum_{\alpha=1}^{m} \kappa_{\alpha} \phi_{\alpha}(t)+\varepsilon \int_{0}^{1} H(t, s) X[s, x(s), \varepsilon] d s\right\| \\
& \leqq \sum_{\alpha=1}^{m} ! \kappa_{\alpha}\left|\cdot\left\|\phi_{\alpha}\right\|_{n}+\right| \varepsilon \mid H_{0} K_{0} \\
& \leqq\left(\sum_{\alpha=1}^{m}\left|\kappa_{\alpha}\right|^{2}\right)^{1 / 2} \cdot\left(\sum_{\alpha=1}^{m} \|\left.\phi_{\alpha}\right|_{n} ^{2}\right)^{1 / 2}+|\varepsilon| H_{0} K_{0} \\
& \leqq\|\kappa\|+\varepsilon_{1} H_{0} K_{0} .
\end{aligned}
$$


Hence we see that $F(x, \kappa ; \varepsilon)$ are certainly defined for

$$
\left\|x-x_{0}\right\|_{n} \leqq \delta_{0}, \quad\|\kappa\| \leqq \delta_{0}-\varepsilon_{1} H_{0} K_{0}
$$

and

$$
|\varepsilon| \leqq \varepsilon_{1}
$$

Here it is needless to say that

$$
\delta_{0}-\varepsilon_{1} H_{0} K_{0}>0
$$

since (3.28) implies

$$
\begin{aligned}
& \varepsilon_{1} \leqq \delta_{0}\left[\left(1+\frac{M}{1-k}\right) H_{0} K_{0}+\frac{L M V W}{1-k}\left(H_{0} K_{0} K_{1}+K_{2}\right)\right]^{-1} \\
& \quad<\delta_{0} /\left(H_{0} K_{0}\right) .
\end{aligned}
$$

(ii) The Fréchet derivative of $F(x, \kappa ; \varepsilon)$ and operator $\hat{J}$. Let $J_{i 1}(x, \kappa ; \varepsilon)$ and $J_{i 2}(x, \kappa ; \varepsilon)(i=1,2)$ be respectively the Fréchet derivatives of $F_{i}(x, \kappa ; \varepsilon)$ with respect to $x$ and $\kappa$, and put

$$
J(x, \kappa ; \varepsilon)=\left[\begin{array}{ll}
J_{11}(x, \kappa ; \varepsilon) & J_{12}(x, \kappa ; \varepsilon) \\
J_{21}(x, \kappa ; \varepsilon) & J_{22}(x, \kappa ; \varepsilon)
\end{array}\right] .
$$

Then evidently $J(x, \kappa ; \varepsilon)$ is the Fréchet derivative of $F(x, \kappa ; \varepsilon)$ with respect to $\{x, \kappa\}$. By our definition, from (3.35), it readily follows that, for any continuous vector function $h(t)$ and any $m$-dimensional vector $\lambda$ whose components are $\lambda_{\beta}(\beta=1,2, \cdots, m)$,

$$
\left\{\begin{array}{l}
J_{11}(x, \kappa ; \varepsilon) h=h(t)-\varepsilon \int_{0}^{1} H(t, s) \Psi[s, x(s), \varepsilon] h(s) d s, \\
J_{12}(x, \kappa ; \varepsilon) \lambda=-\sum_{\beta=1}^{m} \lambda_{\beta} \phi_{\beta}(t), \\
J_{21}(x, \kappa ; \varepsilon) h=\varepsilon \Delta \sum_{i=0}^{N} L_{i} \Phi\left(t_{i}\right) \int_{0}^{t_{t}} \Phi^{-1}(s)\left\{\Psi \left[s, x_{0}(s)+\sum_{\alpha=1}^{m} \kappa_{\alpha} \phi_{\alpha}(s)\right.\right. \\
\left.\left.\quad+\varepsilon \int_{0}^{1} H(s, \sigma) X[\sigma, x(\sigma), \varepsilon] d \sigma, \varepsilon\right] \cdot \int_{0}^{1} H(s, \sigma) \Psi[\sigma, x(\sigma), \varepsilon] h(\sigma) d \sigma\right\} d s, \\
J_{22}(x, \kappa ; \varepsilon) \lambda=\Delta \sum_{i=0}^{N} L_{i} \Phi\left(t_{i}\right) \int_{0}^{t_{i}} \Phi^{-1}(s)\left\{\Psi \left[s, x_{0}(s)+\sum_{\alpha=1}^{m} \kappa_{\alpha} \phi_{\alpha}(s)\right.\right. \\
\left.\left.\quad+\varepsilon \int_{0}^{1} H(s, \sigma) X[\sigma, x(\sigma), \varepsilon] d \sigma, \varepsilon\right] \cdot \sum_{\beta=1}^{m} \lambda_{\beta} \phi_{\beta}(s)\right\} d s .
\end{array}\right.
$$


By the definition of $J_{2}$, it is then clear that

$$
J_{22}\left(x_{0}, 0 ; 0\right)=J_{2} .
$$

Let us take operator $\widehat{J}$ so that

$$
\widehat{J}=J\left(x_{0}, 0 ; 0\right) .
$$

Then by (3.45) and (3.46), the equation

$$
\widehat{J}\{h, \lambda\}=\left\{h^{\prime}, \lambda^{\prime}\right\}
$$

means that

$$
\left\{\begin{array}{l}
h(t)-\sum_{\beta=1}^{m} \lambda_{\beta} \phi_{\beta}(t)=h^{\prime}(t), \\
J_{2} \lambda=\lambda^{\prime},
\end{array}\right.
$$

which can be solved as

$$
\left\{\begin{array}{l}
h(t)=h^{\prime}(t)+\sum_{\beta=1}^{m} \lambda_{\beta} \phi_{\beta}(t), \\
\lambda=J_{2}^{-1} \lambda^{\prime} .
\end{array}\right.
$$

This means that operator $\widehat{J}$ has an inverse $\widehat{J}^{-1}$ and

$$
\{h, \lambda\}=\widehat{J}^{-1}\left\{h^{\prime}, \lambda^{\prime}\right\} .
$$

From the second of (3.49) we have

$$
\|\lambda\| \leqq\left\|J_{2}^{-1}\right\| \cdot\left\|\lambda^{\prime}\right\|
$$

and from the first of (3.49) we have successively

$$
\begin{aligned}
\|h\|_{n} & \leqq\left\|h^{\prime}\right\|_{n}+\sum_{\beta=1}^{m}\left|\lambda_{\beta}\right| \cdot\left\|_{1} \phi_{\beta}\right\|_{n} \\
& \leqq\left\|h^{\prime}\right\|_{n}+\left(\sum_{\beta=1}^{m}\left|\lambda_{\beta}\right|^{2}\right)^{1 / 2} \cdot\left(\sum_{\beta=1}^{m}\left\|\phi_{\beta}\right\|_{n}^{2}\right)^{1 / 2} \\
& =\left\|h^{\prime}\right\|_{n}+\|\lambda\| .
\end{aligned}
$$

Hence, by (3.29), we have

$$
\begin{aligned}
\|\{h, \lambda\}\| & =\|h\|_{n}+\|\lambda\| \\
& \leqq\left\|h^{\prime}\right\|_{n}+2\left\|J_{2}^{-1}\right\| \cdot\left\|\lambda^{\prime}\right\| \\
& \leqq M \cdot\left[\left\|h^{\prime}\right\|_{n}+\left\|\lambda^{\prime}\right\|\right] \\
& =M \cdot\left\|\left\{h^{\prime}, \lambda^{\prime}\right\}\right\|,
\end{aligned}
$$


which by (3.50) implies

$$
\left\|\widehat{J}^{-1}\right\| \leqq M .
$$

This shows that inequality (2.7) in Theorem 2 is valid for $\hat{J}$ defined by (3.47) and $M$ given by (3.29).

(iii) The bound $r$ for the residual error of approximate solution $\left\{x_{0}(t), 0\right\}$. From (3.37) and (3.38), by (3.19), (3.17), (3.8), (3.21) and (3.22), we have

$$
\begin{aligned}
\left\|F\left(x_{0}, 0 ; \varepsilon\right)\right\| & =\left\|F_{1}\left(x_{0}, 0 ; \varepsilon\right)\right\|_{n}+\left\|F_{2}\left(x_{0}, 0 ; \varepsilon\right)\right\| \\
& \leqq|\varepsilon| H_{0} K_{0}+L V W\left(K_{1}|\varepsilon| H_{0} K_{0}+K_{2}|\varepsilon|\right) \\
& =|\varepsilon| \cdot\left[H_{0} K_{0}+L V W\left(H_{0} K_{0} K_{1}+K_{2}\right)\right] .
\end{aligned}
$$

Hence we may suppose that

$$
r=|\varepsilon| \cdot\left[H_{0} K_{0}+L V W\left(H_{0} K_{0} K_{1}+K_{2}\right)\right] .
$$

(iv) The region $D_{\delta}$. We define the region $D_{\delta}$ by

$$
D_{\delta}=\left\{\{x, \kappa\} \mid\left\|x-x_{0}\right\|_{n}+\|\kappa\| \leqq \delta\right\},
$$

where $\delta$ is the number given by (3.33). Since (3.28) implies

$$
\begin{aligned}
& \varepsilon_{1} \leqq \frac{k}{L M V W}\left[\frac { M K _ { 3 } } { 1 - k } \left[H_{0} K_{0}\right.\right.\left.+L V W\left(H_{0} K_{0} K_{1}+K_{2}\right)\right] \\
&\left.+\left(H_{0} K_{0} K_{3}+K_{4}\right)\right]^{-1} \\
&<\frac{k}{L M V W}\left(H_{0} K_{0} K_{3}+K_{4}\right)^{-1},
\end{aligned}
$$

it is clear that

$$
\frac{k}{L M V W}-\varepsilon_{1}\left(H_{0} K_{0} K_{3}+K_{4}\right)>0,
$$

which together with (3.43) implies

$$
\delta>0 \text {. }
$$

Now for any $\{x, \kappa\} \in D_{\delta}$, by (3.33), we have

$$
\left\{\begin{array}{l}
\left\|x-x_{0}\right\|_{n} \leqq \delta \leqq \delta_{0}-\varepsilon_{1} H_{0} K_{0}<\delta_{0}, \\
\|\kappa\| \leqq \delta \leqq \delta_{0}-\varepsilon_{1} H_{0} K_{0} .
\end{array}\right.
$$

This implies that any $\{x, \kappa\} \in D_{\delta}$ satisfies the inequalities (3.41), that 
is, region $D_{\delta}$ is contained in the region of definition of $F(x, \kappa ; \varepsilon)$ for $|\varepsilon| \leqq \varepsilon_{1}$. This means that our $D_{s}$ fulfills condition (2.3) of Theorem 2 for $|\varepsilon| \leqq \varepsilon_{1}$.

(v) Condition (2.4) of Theorem 2. Let $h(t)$ be an arbitrary vector function continuous on $I$ and $\lambda$ be an arbitrary $m$-dimensional vector whose components are $\lambda_{\alpha}(\alpha=1,2, \cdots, m)$. Then by (3.45) and (3. 47), we have

$$
\begin{gathered}
{[J(x, \kappa ; \varepsilon)-\widehat{J}]\{h, \lambda\}} \\
=\left\{-\varepsilon \int_{0}^{1} H(t, s) \Psi[s, x(s), \varepsilon] h(s) d s,\right. \\
\varepsilon \Delta \sum_{i=0}^{N} L_{i} \Phi\left(t_{i}\right) \int_{0}^{t_{i}} \Phi^{-1}(s)\left\{\Psi \left[s, x_{0}(s)+\sum_{\alpha=1}^{m} \kappa_{\alpha} \phi_{\alpha}(s)\right.\right. \\
\left.\left.+\varepsilon \int_{0}^{1} H(s, \sigma) X[\sigma, x(\sigma), \varepsilon] d \sigma, \varepsilon\right] \cdot \int_{0}^{1} H(s, \sigma) \Psi[\sigma, x(\sigma), \varepsilon] h(\sigma) d \sigma\right\} d s \\
+\Delta \sum_{i=0}^{N} L_{i} \Phi\left(t_{i}\right) \int_{0}^{t_{i}} \Phi^{-1}(s)\left\{\Psi \left[s, x_{0}(s)+\sum_{\alpha=1}^{m} \kappa_{\alpha} \phi_{\alpha}(s)\right.\right. \\
\left.\left.+\varepsilon \int_{0}^{1} H(s, \sigma) X[\sigma, x(\sigma), \varepsilon] d \sigma, \varepsilon\right] \cdot \sum_{\beta=1}^{m} \lambda_{\beta} \phi_{\beta}(s)\right\} d s \\
-\Delta \sum_{i=0}^{N} L_{i} \Phi\left(t_{i}\right) \int_{0}^{t_{i}} \Phi^{-1}(s) \Psi\left[s, x_{0}(s), 0\right] \cdot \sum_{\beta=1}^{m} \lambda_{\beta} \phi_{\beta}(s) d s .
\end{gathered}
$$

Hence, if $|\varepsilon| \leqq \varepsilon_{1}$, for $\{x, \kappa\} \in D_{\delta}$, by (3.19), (3.17), (3.8), (3.21), (3.22), (3.18), (3.40) and (3.7), we have

$$
\begin{aligned}
& \|[J(x, \kappa ; \varepsilon)-\widehat{J}]\{h, \lambda\}\| \\
& \leqq|\varepsilon| H_{0} K_{1}\|h\|_{n}+|\varepsilon| L V W K_{1} \cdot H_{0} K_{1}\|h\|_{n} \\
& \quad+L V W\left[K_{3}\left(\|\kappa\|+|\varepsilon| H_{0} K_{0}\right)+K_{4}|\varepsilon|\right]\|\lambda\| \\
& \leqq \varepsilon_{1} H_{0} K_{1}\left(1+L V W K_{1}\right)\|h\|_{n} \\
& \quad+L V W\left[K_{3} \delta+\left(H_{0} K_{0} K_{3}+K_{4}\right) \varepsilon_{1}\right] \cdot\|\lambda\| .
\end{aligned}
$$

However by (3.28),

$$
\varepsilon_{1} H_{0} K_{1}\left(1+L V W K_{1}\right) \leqq k / M,
$$

and by (3.33),

$$
L V W\left[K_{3} \delta+\left(H_{0} K_{0} K_{3}+K_{4}\right) \varepsilon_{1}\right] \leqq k / M .
$$

Hence for $\{x, \kappa\} \in D_{\delta}$ and $|\varepsilon| \leqq \varepsilon_{1}$, from (3.56), we have 


$$
\begin{aligned}
\|[J(x, \kappa ; \varepsilon)-\widehat{J}]\{h, \lambda\}\| & \leqq \frac{k}{M}\left(\|h\|_{n}+\|\lambda\|\right) \\
& =\frac{k}{M} \cdot\|\{h, \lambda\}\|,
\end{aligned}
$$

which clearly implies

$$
\|J(x, \kappa ; \varepsilon)-\widehat{J}\| \leqq k / M
$$

for $\{x, \kappa\} \in D_{\delta}$ and $|\varepsilon| \leqq \varepsilon_{1}$. This shows that condition (2.4) of Theorem 2 is fulfilled for $|\varepsilon| \leqq \varepsilon_{1}$.

(vi) Condition (2.5) of Theorem 2. For $|\varepsilon| \leqq \varepsilon_{1}$, by (3.52) and (3.28), we have

$$
\begin{aligned}
& \frac{M r}{1-k} \leqq \frac{M}{1-k}\left[H_{0} K_{0}+L V W\left(H_{0} K_{0} K_{1}+K_{2}\right)\right] \varepsilon_{1} \\
= & {\left[\left(1+\frac{M}{1-k}\right) H_{0} K_{0}+\frac{L M V W}{1-k}\left(H_{0} K_{0} K_{1}+K_{2}\right)\right] \varepsilon_{1}-H_{0} K_{0} \varepsilon_{1} } \\
& \leqq \\
\quad & \delta_{0}-\varepsilon_{1} H_{0} K_{0},
\end{aligned}
$$

and

$$
\begin{aligned}
\frac{M r}{1-k} \leqq & \frac{M}{1-k}\left[H_{0} K_{0}+L V W\left(H_{0} K_{0} K_{1}+K_{2}\right)\right] \varepsilon_{1} \\
\leqq & \frac{1}{K_{3}} \cdot\left[\left\{\frac{M K_{3}}{1-k}\left[H_{0} K_{0}+L V^{\prime} W\left(H_{0} K_{0} K_{1}+K_{2}\right)\right]\right.\right. \\
& \left.\left.+\left(H_{0} K_{0} K_{3}+K_{4}\right)\right\} \varepsilon_{1}-\left(H_{0} K_{0} K_{3}+K_{4}\right) \varepsilon_{1}\right] \\
\leqq & \frac{1}{K_{3}} \cdot\left[\frac{k}{L M V W}-\left(H_{0} K_{0} K_{3}+K_{\iota}\right) \varepsilon_{1}\right]
\end{aligned}
$$

Hence by (3.33), we see that

$$
\frac{M r}{1-k} \leqq \delta,
$$

which proves that condition (2.5) of Theorem 2 is fulfilled for $|\varepsilon| \leqq \varepsilon_{1}$.

Through (i) $\sim(v i)$, we have seen that for equation (3.36) the conditions of Theorem 2 are all fulfilled by the approximate solution $\left\{x_{0}(t), 0\right\}$ provided $|\varepsilon| \leqq s_{1}$. Thus by the conclusion of Theorem 2 we see that for $|\varepsilon| \leqq \varepsilon_{1}$, equation (3.36) possesses a unique solution $\{x, \kappa\}$ $\left.=\left\{x^{\star}\right), \hat{\kappa}\right\}$ in region $D_{s}$ and moreover there exists $J^{-1}(\hat{x}, \hat{\kappa} ; \varepsilon)$ and 


$$
\left\|\hat{x}-x_{0}\right\|_{n}+\|\hat{\kappa}\| \leqq \frac{M}{1-k}\left[H_{0} K_{0}+L V W\left(H_{0} K_{0} K_{1}+K_{2}\right)\right] \cdot|\varepsilon|
$$

By the remark made in the beginning of the proof, $\hat{x}(t)$ is a solution of boundary value problem (3.1) (3.2). This proves the existence of a solution of the given boundary value problem (3.1) (3.2).

Inequality (3.30) in the conclusion of the theorem readily follows from (3. 57).

We shall now prove the isolatedness and the uniqueness of the solution $x=\hat{x}(t)$.

$1^{\circ}$ Proof of the isolatedness. The first variation equation of (3.1) with respect to $x=\hat{x}(t)$ is

$$
\frac{d h}{d t}=A(t) h+\varepsilon \Psi[t, \hat{x}(t), \varepsilon] h,
$$

and hence for proving the isolatedness of the solution $x=\hat{x}(t)$, it suffices to prove that equation (3.58) possesses no non-trivial solution satisfying

$$
\sum_{i=0}^{N} L_{i} h\left(t_{i}\right)=0
$$

In fact, let $\widehat{\Phi}(t)$ be the fundamental matrix of (3.58) satisfying the initial condition $\widehat{\Phi}(0)=E$. If $x=\hat{x}(t)$ is not isolated, then by the definition of the isolatedness it holds that

$$
\operatorname{det} \sum_{i=0}^{N} L_{i} \widehat{\Phi}\left(t_{i}\right)=0
$$

therefore there is a non-trivial vector $c$ satisfying

$$
\sum_{i=0}^{N} L_{i} \widehat{\Phi}\left(t_{i}\right) \cdot c=0
$$

Put

$$
h(t)=\widehat{\Phi}(t) c
$$

then this is clearly a solution of (3.58) and moreover satisfies (3.59) on account of (3.60). This says that if $x=\hat{x}(t)$ is not isolated, then there is a non-trivial solution of (3.58) satisfying (3.59), in other 
words, if (3.58) possesses no non-trivial solution satisfying (3.59), then $x=\hat{x}(t)$ is isolated.

Now let $h(t)$ be an arbitrary solution of (3.58) satisfying (3.59). Then by Theorem 1 we have

$$
\begin{aligned}
& h(t)=\sum_{\alpha=1}^{m} \lambda_{\alpha} \phi_{\alpha}(t)+\varepsilon \int_{0}^{1} H(t, s) \Psi[s, \hat{x}(s), \varepsilon] h(s) d s, \\
& \Delta \sum_{i=0}^{N} L_{i} \Phi\left(t_{i}\right) \int_{0}^{t_{i}} \Phi^{-1}(s) \Psi[s, \hat{x}(s), \varepsilon] h(s) d s=0
\end{aligned}
$$

for $\varepsilon \neq 0$, where $\lambda_{\alpha}(\alpha=1,2, \cdots, m)$ are arbitrary constants. Rewrite (3.61) as

$$
h(t)-\varepsilon \int_{0}^{1} H(t, s) \Psi[s, \hat{x}(s), \varepsilon] h(s) d s-\sum_{\alpha=1}^{m} \lambda_{\alpha} \phi_{\alpha}(t)=0,
$$

then by (3.45) we have

$$
J_{11}(\hat{x}, \hat{\kappa} ; \varepsilon) h+J_{12}(\hat{x}, \hat{\kappa} ; \varepsilon) \lambda=0,
$$

where $\lambda$ is an $m$-dimensional vector whose components are $\lambda_{\alpha}(\alpha=1,2$, $\cdots, m)$. Next substitute (3.61) into (3.62), then we have

$$
\begin{gathered}
\varepsilon \Delta \sum_{i=0}^{N} L_{i} \Phi\left(t_{i}\right) \int_{0}^{t_{i}} \Phi^{-1}(s) \Psi[s, \hat{x}(s), \varepsilon]\left\{\int_{0}^{1} H(s, \sigma) \Psi[\sigma, \hat{x}(\sigma), \varepsilon] h(\sigma) d \sigma\right\} d s \\
+\Delta \sum_{i=0}^{N} L_{i} \Phi\left(t_{i}\right) \int_{0}^{t_{i}} \Phi^{-1}(s) \Psi[s, \hat{x}(s), \varepsilon] \cdot \sum_{\beta=1}^{m} \lambda_{\beta} \phi_{\beta}(s) \cdot d s=0,
\end{gathered}
$$

which by (3.45) means

$$
J_{21}(\hat{x}, \hat{\kappa} ; \varepsilon) h+J_{22}(\hat{x}, \hat{\kappa} ; \varepsilon) \lambda=0 .
$$

The above equation together with (3.63) implies

$$
J(\hat{x}, \hat{\kappa} ; \varepsilon)\{h, \lambda\}=0 .
$$

Since $J(\hat{x}, \hat{\kappa} ; \varepsilon)$ has an inverse, we thus have

$$
\{h, \lambda\}=0,
$$

that is,

$$
h(t) \equiv 0 \text { and } \lambda_{\alpha}=0 \quad(\alpha=1,2, \cdots, m) .
$$

This proves that equation (3.58) possesses no non-trivial solution satisfying (3.59). By the remark made in the beginning, this implies that 
the solution $x=\hat{x}(t)$ of boundary value problem (3.1) (3.2) is isolated.

$2^{\circ} \quad$ Proof of the uniqueness. Let $x=x(t)$ be an arbitrary solution of the given boundary value problem (3.1) (3.2) lying in region (3.31) for $\varepsilon$ satisfying (3.32).

In (3.31) it is needless to say that

$$
0<\frac{1}{1+K}\left(\delta-|\varepsilon| H_{0} K_{0} K\right)<\delta
$$

for $\varepsilon$ satisfying (3.32).

Now, since $x=x(t)$ is a solution of boundary value problem (3.1) $\sim$ (3.2) for $\varepsilon \neq 0$, equalities (3.34) hold for present $x(t)$. Then from the first of these equalities we have

$$
\sum_{\alpha=1}^{m} \kappa_{\alpha} \phi_{\alpha}(t)=x(t)-x_{0}(t)-\varepsilon \int_{0}^{1} H(t, s) X[s, x(s), \varepsilon] d s .
$$

Then for the $m$-dimensional vector $\kappa$ whose components are $\kappa_{\alpha}(\alpha=1$, $2, \cdots, m)$, by (3.24), (3.31), (3.19) and (3.17), we have

$$
\begin{aligned}
\|\kappa\| & \leqq K\left[\frac{1}{1+K}\left(\delta-|\varepsilon| H_{0} K_{0} K\right)+|\varepsilon| H_{0} K_{0}\right] \\
& =\frac{K}{1+K}\left(\delta+|\varepsilon| H_{0} K_{0}\right) .
\end{aligned}
$$

Then by (3.31) we have

$$
\begin{aligned}
\left\|x-x_{0}\right\|_{n}+\|\kappa\| & \leqq \frac{1}{1+K}\left(\delta-|\varepsilon| H_{0} K_{0} K\right)+\frac{K}{1+K}\left(\delta+|\varepsilon| H_{0} K_{0}\right) \\
& =\delta
\end{aligned}
$$

which means that $\{x(t), \kappa\} \in D_{\delta}$. Since $\{x(t), \kappa\}$ is a solution of (3.36) and (3.36) possesses a unique solution $\{\hat{x}(t), \hat{\kappa}\}$ in $D_{\S}$ for $|\varepsilon| \leqq \varepsilon_{1}$, we thus have

$$
x(t)=\hat{x}(t), \quad \kappa=\hat{\kappa} .
$$

This proves the uniqueness of the solution of boundary value problem (3.1) (3.2) in region (3.31) for $\varepsilon$ satisfying (3.32) and this completes the proof of the theorem.

Q. E. D.

Remark. The solution $x=\hat{x}(t)$ obtained in Theorem 3 is isolated 
and hence it will be possible to compute such a solution on a machine starting from the approximate solution $x=x_{0}(t)$ if one uses, say, the method developed by the author in [3].

3.4. An example. Theorem 3 will be illustrated with the boundary value problem:

(3.65) $\ddot{x}+4 \pi^{2} x=e(t)+\varepsilon f(t, x, \dot{x}, \varepsilon) \quad(\cdot=d / d t)$,

(3.66) $\quad x(0)=l_{1}, \quad x(1)=l_{2}$.

Put

$$
x=x_{1}, \quad \dot{x}=2 \pi x_{2},
$$

then corresponding to (3.65) and (3.66), we have

(3. 68) $\left\{\begin{array}{l}\dot{x}_{1}=2 \pi x_{2}, \\ \dot{x}_{2}=-2 \pi x_{1}+\frac{1}{2 \pi} e(t)+\frac{\varepsilon}{2 \pi} f\left(t, x_{1}, 2 \pi x_{2}, \varepsilon\right),\end{array}\right.$

$$
x_{1}(0)=l_{1}, \quad x_{1}(1)=l_{2} .
$$

Comparing these with $(3.1) \sim(3.2)$, we see that

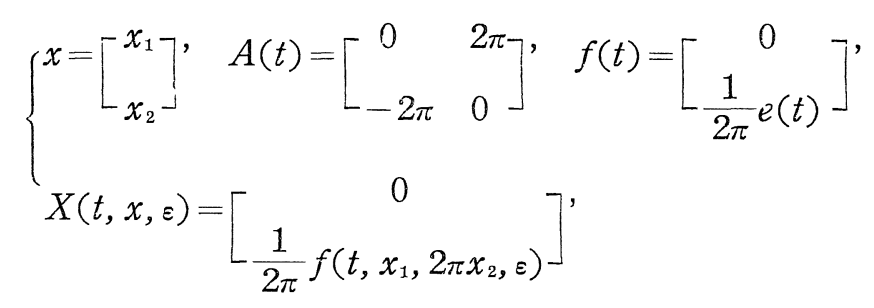

(3. 71) $\begin{cases}N=1, & t_{0}=0, \quad t_{1}=1, \\ L_{0}=\left[\begin{array}{ll}1 & 0 \\ 0 & 0\end{array}\right], & L_{1}=\left[\begin{array}{ll}0 & 0 \\ 1 & 0\end{array}\right], \quad l=\left[\begin{array}{l}l_{1} \\ l_{2}\end{array}\right] .\end{cases}$

By (3. 70),

$$
\left\{\begin{array}{rr}
\Phi(t) & =\left[\begin{array}{rr}
\cos 2 \pi t & \sin 2 \pi t \\
-\sin 2 \pi t & \cos 2 \pi t
\end{array}\right] \\
\Phi^{-1}(t) & =\left[\begin{array}{rr}
\cos 2 \pi t & -\sin 2 \pi t \\
\sin 2 \pi t & \cos 2 \pi t
\end{array}\right]
\end{array}\right.
$$

consequently 


$$
G=\sum_{i=0}^{1} L_{i} \Phi\left(t_{i}\right)=\left[\begin{array}{ll}
1 & 0 \\
1 & c
\end{array}\right] .
$$

Then $m=1$ and we may take $\phi_{1}(t), d_{1}$ and $S$ so that

$$
\phi_{1}(t)=\left[\begin{array}{c}
\sin 2 \pi^{t} \\
\cos 2 \pi t
\end{array}\right], \quad d_{1}=\left[\frac{1}{\sqrt{2}},-\frac{1}{\sqrt{2}}\right],
$$

$$
S=\left[\begin{array}{ll}
1 & 0 \\
0 & 0
\end{array}\right]
$$

Then we readily see that

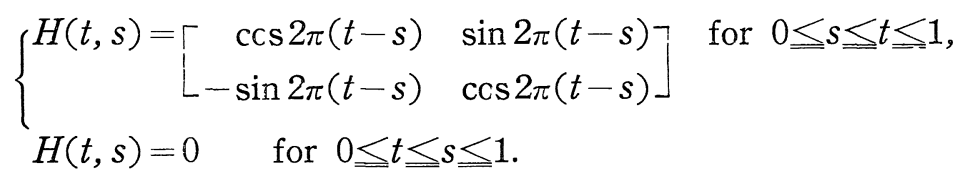

Thus, in the present example, we find after elementary calculations that

$1^{\circ}$ condition (3.9) of Theorem 3 is

$$
l_{1}-l_{2}=\frac{1}{2 \pi} \int_{0}^{1} e(t) \sin 2 \pi t d t,
$$

$2^{\circ} \quad x_{0}(t)$ given by (3.15) is of the form

$$
x_{0}(t)=\left[\begin{array}{l}
\kappa^{0} \sin 2 \pi t+l_{1} \cos 2 \pi t+\frac{1}{2 \pi} \int_{0}^{t} \sin 2 \pi(t-s) \cdot e(s) d s \\
\kappa^{0} \cos 2 \pi t-l_{1} \sin 2 \pi t+\frac{1}{2 \pi} \int_{0}^{t} \cos 2 \pi(t-s) \cdot e(s) d s
\end{array}\right],
$$

$3^{\circ}$ equation (3.14) of Theorem 3 is

$$
\begin{aligned}
& \int_{0}^{1} f\left[t, \kappa \sin 2 \pi t+l_{1} \cos 2 \pi t+\frac{1}{2 \pi} \int_{0}^{t} \sin 2 \pi(t-s) \cdot e(s) d s,\right. \\
& \left.2 \pi \kappa \cos 2 \pi t-2 \pi l_{1} \sin 2 \pi t+\int_{0}^{t} \operatorname{ccs} 2 \pi(t-s) \cdot e(s) d s, 0\right] \sin 2 \pi t d t \\
= & 0 .
\end{aligned}
$$

Hence by Theorem 3 we see that if $f(t, x, \dot{x}, \varepsilon)$ is twice continuously differentiable with respect to $x, \dot{x}$ and $\varepsilon$, equality (3.77) is valid for $l_{1}, l_{2}$ and $e(t)$, and there is $\kappa=\kappa^{0}$ satisfying (3.79) such that the de- 
rivative of the left member of (3.79) with respect to $\kappa$ dces not vanish for $\kappa=\kappa^{0}$, then for sufficiently small $|\varepsilon|$, given boundary value problem (3.65) (3.66) possesses a unique isolated solution $x=\hat{x}(t)$ which converges to $x_{0}(t)$ given by (3.78) as $\varepsilon \rightarrow 0$.

If we replace boundary condition (3.66) by

$$
x(0)=x(1), \quad \dot{x}(0)=\dot{x}(1),
$$

then instead of (3.71), we have

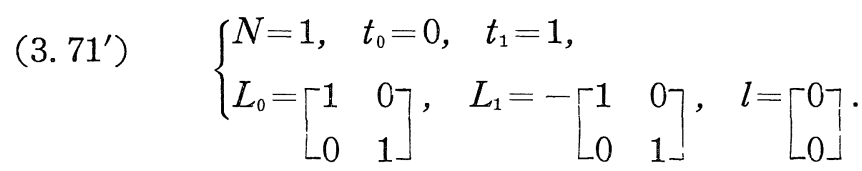

Hence instead of (3.73), (3.74) and (3.75), we have respectively

$$
G=0 \text {; }
$$

(3. $74^{\prime}$ )

$$
\begin{aligned}
\phi_{1}(t) & =\frac{1}{\sqrt{2}}\left[\begin{array}{r}
\cos 2 \pi t \\
-\sin 2 \pi t
\end{array}\right], \quad \phi_{2}(t)=\frac{1}{\sqrt{2}}\left[\begin{array}{l}
\sin 2 \pi t \\
\cos 2 \pi t
\end{array}\right] ; \\
d_{1} & =\left[\frac{1}{\sqrt{2}}, 0\right], \quad d_{2}=\left[0, \frac{1}{\sqrt{2}}\right] ;
\end{aligned}
$$

(3. $\left.75^{\prime}\right)$

$$
S=\left[\begin{array}{ll}
0 & 0 \\
0 & 0
\end{array}\right] \text {. }
$$

Thus, in the present case, we find that

$$
1^{\circ} \text { condition (3.9) of Theorem } 3 \text { is }
$$

$$
\int_{0}^{1} e(t)\left[\begin{array}{c}
\sin 2 \pi t \\
\cos 2 \pi t
\end{array}\right] d t=0
$$

$2^{\circ} \quad x_{0}(t)$ given by (3.15) is

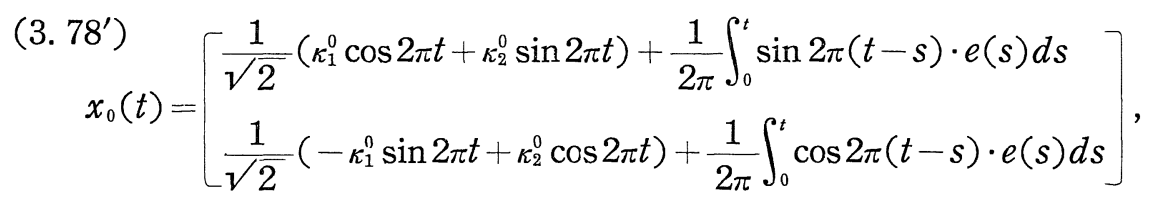

$3^{\circ}$ equation (3.14) of Theorem 3 is

(3. $\left.79^{\prime}\right)$

$$
\int_{0}^{1} f \cdot\left[\begin{array}{c}
\sin 2 \pi t \\
\cos 2 \pi t
\end{array}\right] d t=0
$$


where

(3. 80) $f=f\left[t, \frac{1}{\sqrt{2}}\left(\kappa_{1} \cos 2 \pi t+\kappa_{2} \sin 2 \pi t\right)+\frac{1}{2 \pi} \int_{0}^{t} \sin 2 \pi(t-s) \cdot e(s) d s\right.$, $\left.\frac{2 \pi}{\sqrt{2}}\left(-\kappa_{1} \sin 2 \pi t+\kappa_{2} \cos 2 \pi t\right)+\int_{0}^{t} \cos 2 \pi(t-s) \cdot e(s) d s, 0\right]$.

Hence by Theorem 3 we see that if $f(t, x, \dot{x}, \varepsilon)$ is twice continuously differentiable with respect to $x, \dot{x}$ and $\varepsilon$, equality $\left(3.77^{\prime}\right)$ is valid for $e(t)$, and there are $\kappa_{\alpha}=\kappa_{\alpha}^{0}(\alpha=1,2)$ satisfying $\left(3.79^{\prime}\right)$ such that the Jacobian of the left member of $\left(3.79^{\prime}\right)$ with respect to $\kappa_{\alpha}(\alpha=1,2)$ does not vanish for $\kappa_{\alpha}=\kappa_{\alpha}^{0}(\alpha=1,2)$, then for sufficiently small $|\varepsilon|$, given boundary value problem (3.65) and $\left(3.66^{\prime}\right)$ possesses a unique isolated solution $x=\hat{x}(t)$ which converges to $x_{0}(t)$ given by $\left(3.78^{\prime}\right)$ as $\varepsilon \rightarrow 0$.

\section{§4. Application to Boundary Value Problems Associated with Nonlinear Differential Systems Containing a Small Parameter}

Nonlinear differential systems containing a small parameter can be written in the form (0.6). In the present paragraph, by the application of Theorem 3 the following boundary value problem will be solved:

$$
\begin{aligned}
& \frac{d \xi}{d t}=\Xi(t, \xi)+\varepsilon \Theta(t, \xi, \varepsilon), \\
& \sum_{i=0}^{N} L_{i} \xi\left(t_{i}\right)=l,
\end{aligned}
$$

where $\xi, \Xi(t, \xi)$ and $\Theta(t, \xi, \varepsilon)$ are $n$-dimensional vectors, $\varepsilon$ is a parameter, $L_{i}(i=0,1,2, \cdots, N)$ are given $n \times n$ matrices, $l$ is a given $n$-dimensional vector, and

$$
0=t_{0}<t_{1}<t_{2}<\cdots<t_{N-1}<t_{N}=1
$$

4. 1. Preliminaries. In (4.1) we suppose that $\Xi(t, \xi)$ is four times continuously differentiable with respect to $\xi$ and $\Theta(t, \xi, \varepsilon)$ is three times continuously differentiable with respect to $\xi$ and $\varepsilon$ for $(t, \xi) \in \Omega$ and $|\varepsilon| \leqq \varepsilon_{0}$, where $\varepsilon_{0}$ is a positive number and $\Omega$ is the domain of the $t \xi$ - 
space intercested by two hyperplanes $t=0$ and $t=1$ such that every section of $\Omega$ by an arbitrary hyperplane $t=\tau(0 \leqq \tau \leqq 1)$ is a non-empty open set of the $\xi$-space.

We assume that the unperturbed system of (4.1)

$$
\frac{d z}{d t}=\Xi(t, z)
$$

possesses a solution $z=\xi_{0}(t)$ satis 1 ying boundary condition (4.2) such that the graph of $\xi=\xi_{0}(t)$ lies in $\Omega$ for $0 \leqq t \leqq 1$. By the assumption on $\Omega$, it is clear that there is a positive number $\delta_{0}$ such that

$$
\Omega_{0}=\left\{(t, \xi)\|\| \xi-\xi_{0}(t) \| \leqq \delta_{0}, t \in I[0,1]\right\} \subset \Omega .
$$

Let $\Psi(t, \xi)$ be the Jacobian matrix of $\Xi(t, \xi)$ with respect to $\xi$ and put

$$
\Psi\left[t, \xi_{0}(t)\right]=A(t) .
$$

Then the linear differential system

$$
\frac{d y}{d t}=A(t) y
$$

is the first variation equation of (4.3) with respect to the solution $z=\xi_{0}(t)$. Let $\Phi(t)$ be the fundamental matrix of (4.6) satisfying the initial condition $\Phi(0)=E$. When the matrix

$$
G=\sum_{i=0}^{N} L_{i} \Phi\left(t_{i}\right)
$$

is non-singular, that is, the solution $z=\xi_{0}(t)$ is isolated, boundary value problem $(4.1) \sim(4.2)$ has been already solved in [1]. Hence in the present paragraph the case where $G$ is singular will be discussed.

Suppose that the rank of $G$ is $n-m(1 \leqq m \leqq n)$. Then according to Theorem 1 , we have $m$ linearly independent solutions $\phi_{\alpha}(t)(\alpha=1,2$, $\cdots, m)$ of (4.6) satisfying the boundary condition

$$
\sum_{i=0}^{N} L_{i} y\left(t_{i}\right)=0
$$

$m$ linearly independent vectors $d_{\alpha}(\alpha=1,2, \cdots, m)$ satisfying 


$$
d_{\alpha} G=0 \text {, }
$$

and the matrix $H(t, s)$ of the $H$-mapping corresponding to matrices $A(t)$ and $L_{i}(i=0,1,2, \cdots, N)$.

The symbols necessary for succeeding discussions will be now introduced.

$1^{\circ} \Gamma(t, \xi), \Theta_{1}(t, \xi, \varepsilon)$ and $\Theta_{2}(t, \xi, \varepsilon) . \quad \Gamma(t, \xi)$ denotes the Fréchet derivative of $\Psi(t, \xi)$ with respect to $\xi, \Theta_{1}(t, \xi, \varepsilon)$ denotes the Jacobian matrix of $\Theta(t, \xi, \varepsilon)$ with respect to $\xi$, and $\Theta_{2}(t, \xi, \varepsilon)$ denotes the derivative of $\Theta(t, \xi, \varepsilon)$ with respect to $\varepsilon$.

$2^{\circ} \Delta$. $\Delta$ denotes the matrix whose row vectors are $d_{\alpha}(\alpha=1,2$, $\cdots, m)$.

$3^{\circ} \theta_{0}, C_{\alpha \beta}, C_{\alpha}$ and $C_{0}(\alpha, \beta=1,2, \cdots, m) . \quad$ These symbols denote respectively the following $m$-dimensional constant vectors:

$$
\left\{\begin{array}{l}
\Theta_{0}=\Delta \sum_{i=0}^{N} L_{i} \Phi\left(t_{i}\right) \int_{0}^{t_{i}} \Phi^{-1}(t) \Theta\left[t, \xi_{0}(t), 0\right] d t, \\
C_{\alpha \beta}=\Delta \sum_{i=0}^{N} L_{i} \Phi\left(t_{i}\right) \int_{0}^{t_{t}} \Phi^{-1}(t) \Gamma\left[t, \xi_{0}(t)\right] \phi_{\alpha}(t) \phi_{\beta}(t) d t, \\
C_{\alpha}=\Delta \sum_{i=0}^{N} L_{i} \Phi\left(t_{i}\right) \int_{0}^{t_{t}} \Phi^{-1}(t)\left\{\Gamma\left[t, \xi_{0}(t)\right] \int_{0}^{1} H(t, s) \Theta\left[s, \xi_{0}(s), 0\right] d s\right. \\
\left.\quad+\Theta_{1}\left[t, \xi_{0}(t), 0\right]\right\} \phi_{\alpha}(t) d t, \\
\quad C_{0}=\Delta \sum_{i=0}^{N} L_{i} \Phi\left(t_{i}\right) \int_{0}^{t_{i}} \Phi^{-1}(t)\left\{\Gamma\left[t, \xi_{0}(t)\right]\right. \\
\quad \times \int_{0}^{1} H(t, s) \Theta\left[s, \xi_{0}(s), 0\right] d s \cdot \int_{0}^{1} H(t, s) \Theta\left[s, \xi_{0}(s), 0\right] d s \\
\quad+2 \Theta_{1}\left[t, \xi_{0}(t), 0\right] \cdot \int_{0}^{1} H(t, s) \Theta\left[s, \xi_{0}(s), 0\right] d s \\
\left.\quad+2 \Theta_{2}\left[t, \xi_{0}(t), 0\right]\right\} d t \quad(\alpha, \beta=1,2, \cdots, m) .
\end{array}\right.
$$

4. 2. Theorem concerning boundary value problem $(4.1) \sim(4.2)$.

Theorem 4. For boundary value problem (4.1) (4.2), assume that the appearing functions have the smoothness mentioned in the preceding section and that the rank of matrix $G$ defined by (4.7) is $n-m(1 \leqq m \leqq n)$. 
If $\Theta_{0}=0$ and the equation

$$
\sum_{\alpha, \beta=1}^{m} C_{\alpha \beta} \kappa_{\alpha} \kappa_{\beta}+2 \sum_{\alpha=1}^{m} C_{\alpha} \kappa_{\alpha}+C_{0}=0
$$

possesses a solution $\kappa_{\alpha}=\kappa_{\alpha}^{0}(\alpha=1,2, \cdots, m)$ for which the Jacobian of the left member of (4.9) does not vanish, then boundary value problem (4.1) (4.2) possesses a unique isolated solution $\xi=\hat{\xi}(t)$ in the neighborhood of

(4. 10) $\bar{\xi}(t)=\xi_{0}(t)+\varepsilon\left\{\sum_{\alpha=1}^{m} \kappa_{\alpha}^{0} \phi_{\alpha}(t)+\int_{0}^{1} H(t, s) \Theta\left[s, \xi_{0}(s), 0\right] d s\right\}$

for sufficiently small $|\varepsilon|>0$, and for such $\hat{\xi}(t)$, it holds that

$$
\|\hat{\xi}(t)-\bar{\xi}(t)\|_{n}=O\left(\varepsilon^{2}\right) \quad(\varepsilon \rightarrow 0) .
$$

If $\Theta_{0} \neq 0$ and the equation

$$
\sum_{\alpha, \beta=1}^{n} C_{\alpha \beta} \kappa_{\alpha} \kappa_{\beta}+2 \Theta_{0}=0
$$

possesses a solution $\kappa_{\alpha}=\kappa_{\alpha}^{0}(\alpha=1,2, \cdots, m)$ for which the Jacobian of the left member of (4.12) does not vanish, then boundary value problem (4.1) (4.2) possesses a unique isolated solution $\xi=\hat{\xi}(t)$ in the neighborhood of

$$
\bar{\xi}(t)=\xi_{0}(t)+\varepsilon^{1 / 2} \sum_{\alpha=1}^{m} \kappa_{\alpha}^{0} \phi_{\alpha}(t)
$$

for sufficiently small $|\varepsilon|>0$, and for such $\hat{\xi}(t)$, is holds that

$$
\|\hat{\xi}(t)-\bar{\xi}(t)\|_{n}=O(\varepsilon) \quad(\varepsilon \rightarrow 0) \text {. }
$$

Proof. Put

$$
\xi=\xi_{0}(t)+\varepsilon^{\nu} x \quad(\nu=1 \text { or } 1 / 2)
$$

and suppose that

$$
\left|\varepsilon^{\nu}\right| \cdot\|x\| \leq \delta_{0} .
$$

Then substituting (4.15) into (4.1), we have

$$
\begin{aligned}
\frac{d x}{d t}= & \varepsilon^{-\nu}\left\{\Xi\left[t, \xi_{0}(t)+\varepsilon^{\nu} x\right]-\Xi\left[t, \xi_{0}(t)\right]\right\} \\
& +\varepsilon^{1-\nu} \Theta\left[t, \xi_{0}(t)+\varepsilon^{\nu} x, \varepsilon\right] \quad(\varepsilon \neq 0),
\end{aligned}
$$


However by the mean value theorem, we have

$$
\begin{aligned}
& \varepsilon^{-\nu}\left\{\Xi\left[t, \xi_{0}(t)+\varepsilon^{\nu} x\right]-\Xi\left[t, \xi_{0}(t)\right]\right\} \\
= & \int_{0}^{1} \Psi\left[t, \xi_{0}(t)+\theta \varepsilon^{\nu} x\right] d \theta \cdot x \\
= & \Psi\left[t, \xi_{0}(t)\right] x+\int_{0}^{1}\left\{\Psi\left[t, \xi_{0}(t)+\theta \varepsilon^{\nu} x\right]-\Psi\left[t, \xi_{0}(t)\right]\right\} d \theta \cdot x \\
= & A(t) x+\int_{0}^{1}\left\{\int_{0}^{1} \Gamma\left[t, \xi_{0}(t)+\theta_{1} \theta \varepsilon^{\nu} x\right] \theta \varepsilon^{\nu} x d \theta_{1}\right\} d \theta \cdot x \\
= & A(t) x+\varepsilon^{\nu} \int_{0}^{1} \int_{0}^{\theta} \Gamma\left[t, \xi_{0}(t)+\theta_{1} \varepsilon^{\nu} x\right] d \theta_{1} d \theta \cdot x \cdot x .
\end{aligned}
$$

Hence we can rewrite (4.17) as follows:

$$
\begin{aligned}
\frac{d x}{d t}=A(t) x & +\varepsilon^{\nu} \int_{0}^{1} \int_{0}^{\theta} \Gamma\left[t, \xi_{0}(t)+\theta_{1} \varepsilon^{\nu} x\right] d \theta_{1} d \theta \cdot x \cdot x \\
& +\varepsilon^{1-\nu} \Theta\left[t, \xi_{0}(t)+\varepsilon^{\nu} x, \varepsilon\right] .
\end{aligned}
$$

Now let us consider the case where $\nu=1$. In this case, (4.18) is of the form

$$
\frac{d x}{d t}=A(t) x+\Theta\left[t, \xi_{0}(t), 0\right]+\varepsilon X(t, x, \varepsilon),
$$

where

$$
\begin{aligned}
& X(t, x, \varepsilon)=\int_{0}^{1} \int_{0}^{\theta} \Gamma\left[t, \xi_{0}(t)+\theta_{1} \varepsilon x\right] d \theta_{1} d \theta \cdot x \cdot x \\
& \quad+\varepsilon^{-1}\left\{\Theta\left[t, \xi_{0}(t)+\varepsilon x, \varepsilon\right]-\Theta\left[t, \xi_{0}(t), 0\right]\right\} \\
& =\int_{0}^{1} \int_{0}^{\theta} \Gamma\left[t, \xi_{0}(t)+\theta_{1} \varepsilon x\right] d \theta_{1} d \theta \cdot x \cdot x \\
& \quad+\int_{0}^{1} \Theta_{1}\left[t, \xi_{0}(t)+\theta \varepsilon x, \theta \varepsilon\right] d \theta \cdot x+\int_{0}^{1} \Theta_{2}\left[t, \xi_{0}(t)+\theta \varepsilon x, \theta \varepsilon\right] d \theta .
\end{aligned}
$$

Equation (4.19) is of the form (3.1) and moreover, for the solution $\xi=\xi(t)$ of boundary value problem (4.1) (4.2) with $\varepsilon \neq 0$, from (4. 15), we have

$$
\sum_{i=0}^{N} L_{i} x\left(t_{i}\right)=0
$$

Since $X(t, x, \varepsilon)$ is twice continuously differentiable with respect to $x$ and $\varepsilon$ from our assumption, we can now apply Theorem 3 to the weakly nonlinear differential system (4.19) with boundary condition (4.21). 
For this boundary value problem, as seen from (4.8), condition (3.9) of Theorem 3 is

$$
\Theta_{0}=0
$$

and equation (3.14) of Theorem 3 is

$$
\begin{aligned}
\Delta \sum_{i=0}^{N} & L_{i} \Phi\left(t_{i}\right) \int_{0}^{t_{i}} \Phi^{-1}(t) \\
& \times\left\{\frac{1}{2} \Gamma\left[t, \xi_{0}(t)\right] \cdot\left[\sum_{\alpha=1}^{m} \kappa_{\alpha} \phi_{\alpha}(t)+\int_{0}^{1} H(t, s) \Theta\left[s, \xi_{0}(s), 0\right] d s\right]\right. \\
& \times\left[\sum_{\beta=1}^{m} \kappa_{\beta} \phi_{\beta}(t)+\int_{0}^{1} H(t, s) \Theta\left[s, \xi_{0}(s), 0\right] d s\right] \\
& +\Theta_{1}\left[t, \xi_{0}(t), 0\right] \cdot\left[\sum_{\alpha=1}^{m} \kappa_{\alpha} \phi_{\alpha}(t)+\int_{0}^{1} H(t, s) \Theta\left[s, \xi_{0}(s), 0\right] d s\right] \\
& \left.+\Theta_{2}\left[t, \xi_{0}(t), 0\right]\right\} d t=0,
\end{aligned}
$$

which, by (4.8), can be written as

$$
\sum_{\alpha, \beta=1}^{m} C_{\alpha \beta} \kappa_{\alpha} \kappa_{\beta}+2 \sum_{\alpha=1}^{m} C_{a} \kappa_{\alpha}+C_{0}=0 .
$$

Hence by Theorem 3 we get the first half of the theorem except for the isolatedness of the solution $\xi=\hat{\xi}(t)$.

To prove the isolatedness of the solution $\xi=\hat{\xi}(t)$, consider the first variation equation of (4.1) with respect to the solution $\xi=\hat{\xi}(t)$. As readily seen, it reads

$$
\frac{d \eta}{d t}=\left\{\Psi[t, \hat{\xi}(t)]+\varepsilon \Theta_{1}[t, \hat{\xi}(t), \varepsilon]\right\} \eta
$$

On the other hand, as seen from (4.17), the first variation equation of (4.19) with respect to its solution $x=\hat{x}(t)$ is

$$
\frac{d y}{d t}=\left\{\Psi\left[t, \xi_{0}(t)+\varepsilon \hat{x}(t)\right]+\varepsilon \Theta_{1}\left[t, \xi_{0}(t)+\varepsilon \hat{x}(t), \varepsilon\right]\right\} y .
$$

Equation (4.22) then coincides with equation (4.23) since $\hat{\xi}(t)$ and $\hat{x}(t)$ are connected by

$$
\widehat{\xi}(t)=\xi_{0}(t)+\varepsilon \hat{x}(t)
$$

as seen from (4.15). Now by Theorem 3 the solution $x=\hat{x}(t)$ of 
(4.19) is isolated in the case under consideration. Hence we see that the solution $\xi=\widehat{\xi}(t)$ of the given boundary value problem $(4.1) \sim(4.2)$ is also isolated. This completes the proof of the first half of the theorem.

To prove the latter half of the theorem, let us consider the case where $\nu=1 / 2$ in (4.18). In this case, putting

$$
\varepsilon^{1 / 2}=\mu,
$$

we can rewrite $(4.18)$ in the form

$$
\frac{d x}{d t}=A(t) x+\mu X(t, x, \mu),
$$

where

$$
\begin{aligned}
X(t, x, \mu)=\int_{0}^{1} \int_{0}^{\theta} \Gamma\left[t, \xi_{0}(t)\right. & \left.+\theta_{1} \mu x\right] d \theta_{1} d \theta \cdot x \cdot x \\
& +\Theta\left[t, \xi_{0}(t)+\mu x, \mu^{2}\right] .
\end{aligned}
$$

In this case, condition (3.9) of Theorem 3 becomes an identity and equation (3.14) of Theorem 3 becomes

$$
\begin{aligned}
\Delta \sum_{i=0}^{N} L_{i} \Phi\left(t_{i}\right) \int_{0}^{t_{i}} \Phi^{-1}(t)\left\{\frac{1}{2} \Gamma\left[t, \xi_{0}(t)\right] \cdot\left[\sum_{\alpha=1}^{m} \kappa_{\alpha} \phi_{\alpha}(t)\right] \cdot\left[\sum_{\beta=1}^{m} \kappa_{\beta} \phi_{\beta}(t)\right]\right. \\
\left.+\Theta\left[t, \xi_{0}(t), 0\right]\right\} d t=0
\end{aligned}
$$

which, by (4.8), can be written as

$$
\sum_{\alpha, \beta=1}^{m} C_{\alpha \beta} \kappa_{\alpha} \kappa_{\beta}+2 \theta_{0}=0
$$

Thus, in a similar way as before, we get the latter half of the theorem. This completes the proof.

Q. E. D.

Remark 1. Theorem 4 is an extension of Theorem 3. To clarify the relationship between these theorems, consider the case where $\Xi(t, \xi)$ is linear in $\xi$, that is, $\Xi(t, \xi)$ is of the form

$$
\Xi(t, \xi)=A(t) \xi+f(t) \text {. }
$$

Then by Theorem 1 the existence of a solution $z=\xi_{0}(t)$ of (4.3) implies the validity of condition (3.9) of Theorem 3 and vice versa. Moreover by Theorem 1 solution $z=\xi_{0}(t)$ is of the form 


$$
\xi_{0}(t)=\sum_{\alpha=1}^{m} \kappa_{\alpha} \phi_{\alpha}(t)+\Phi(t) S l+\int_{0}^{1} H(t, s) f(s) d s,
$$

where $\kappa_{\alpha}(\alpha=1,2, \cdots, m)$ are constants. Condition " $\Theta_{0}=0$ " of Theorem 4 means then

$$
\begin{gathered}
\Delta \sum_{i=0}^{N} L_{i} \Phi\left(t_{i}\right) \int_{0}^{t_{i}} \Phi^{-1}(t) \Theta\left[t, \sum_{\alpha=1}^{m} \kappa_{\alpha} \phi_{\alpha}(t)+\Phi(t) S l\right. \\
\left.+\int_{0}^{1} H(t, s) f(s) d s, 0\right] d t=0
\end{gathered}
$$

which is nothing else equation (3.14) of Theorem 3.

When $\Xi(t, \xi)$ is linear in $\xi$, it is evident that $\Gamma(t, \xi) \equiv 0$. Hence $C_{\alpha \beta}=0(\alpha, \beta=1,2, \cdots, m)$ and

$$
\begin{gathered}
C_{\alpha}=\Delta \sum_{i=0}^{N} L_{i} \Phi\left(t_{i}\right) \int_{0}^{t_{i}} \Phi^{-1}(t) \Theta_{1}\left[t, \xi_{0}(t), 0\right] \phi_{\alpha}(t) d t \\
(\alpha=1,2, \cdots, m) .
\end{gathered}
$$

Then equation (4.9) of Theorem 4 becomes

$$
2 \sum_{\alpha=1}^{m} C_{\alpha} \kappa_{\alpha}+C_{0}=0
$$

and the non-vanishing of the Jacobian of the left member of equation (4.27) with respect to $\kappa_{\alpha}(\alpha=1,2, \cdots, m)$ means

$$
\operatorname{det}\left[C_{1}, C_{2}, \cdots, C_{m}\right] \neq 0 \text {, }
$$

which evidently implies the existence of a solution of equation (4.29) with non-vanishing Jacobian of the left member of the equation.

The above discussions show that if $\Xi(t, \xi)$ is linear in $\xi$, then the conditions of Theorem 3 imply the conditions of the first half of Theorem 4 except for the smoothness condition on function $\Theta(t, \xi, \varepsilon)$. This shows that Theorem 4 is really an extension of Theorem 3 to general nonlinear differential systems containing a small parameter.

Remark 2. In Theorem 3, as seen from (3.15) and (3.30), the zero-th approximation of the desired solution is given, while in Theorem 4 , as seen from $(4.10) \sim(4.11)$ and $(4.13) \sim(4.14)$, the first approximation of the desired solution is given. 
Remark 3. Equations (4.9) and (4.12) may have several solutions for which the Jacobian of each left member of the equations does not vanish. In such a case, it is needless to say that the given boundary value problem (4.1) (4.2) also possesses several solutions corresponding to solutions of (4.9) and (4.12).

Remark 4. In Theorem 4, the explicit bounds for $|\varepsilon|$ and $\| \hat{\xi}(t)$ $-\bar{\xi}(t) \|_{n}$ are omitted for brevity of the statement. However it is needless to say that they can be obtained, if necessary, by applying Theorem 3 to equations (4.19) and (4.25).

Remark 5. The solutions $\xi=\hat{\xi}(t)$ obtained in Theorem 4 are isolated and hence, like the solution $x=\hat{x}(t)$ obtained in Theorem 3 , it will be possible to compute such solutions on a machine starting from the approximate solutions $\xi=\bar{\xi}(t)$ given by (4.10) and (4.13) if one uses, say, the method developed by the author in [3].

\section{References}

[1] Urabe, M., An existence theorem for multi-point boundary value problems. Funkcial. Ekvac. 9 (1966), 43-60.

[2] — The Newton method and its application to boundary value problems with nonlinear boundary conditions, Proc. US-Japan Seminar on Differential and Functional Equations. Benjamin, New York. 1967, pp. 383-410.

[3] - Numerical solution of multi-point boundary value problems in Chebyshev series-Theory of the method, Numer. Math. 9 (1967). 311-366. 